\title{
A systematic study of mass spectra and strong decay of strange mesons
}

\author{
Cheng-Qun Pang ${ }^{1,2}$, Jun-Zhang Wang ${ }^{1,3}$, Xiang Liu ${ }^{1,3, a}{ }_{\mathbb{C}}$, Takayuki Matsuki ${ }^{4,5, b}$ \\ ${ }^{1}$ School of Physical Science and Technology, Lanzhou University, Lanzhou 730000, China \\ ${ }^{2}$ College of Physics and Electronic Information Engineering, Qinghai Normal University, Xining 810000, China \\ ${ }^{3}$ Research Center for Hadron and CSR Physics, Lanzhou University and Institute of Modern Physics of CAS, Lanzhou 730000, China \\ ${ }^{4}$ Tokyo Kasei University, 1-18-1 Kaga, Itabashi, Tokyo 173-8602, Japan \\ 5 Theoretical Research Division, Nishina Center, RIKEN, Saitama 351-0198, Japan
}

Received: 29 August 2017 / Accepted: 29 November 2017 / Published online: 13 December 2017

(C) The Author(s) 2017. This article is an open access publication

\begin{abstract}
The mass spectrum of the kaon family is analyzed by the modified Godfrey-Isgur model with a color screening effect approximating the kaon as a heavy-light meson system. This analysis gives us the structure and possible assignments of the observed kaon candidates, which can be tested by comparing the theoretical results of their two-body strong decays with the experimental data. Additionally, prediction of some partial decay widths is made on the kaons still missing in experiment. This study is crucial to establishing the kaon family and searching for their higher excitations in the future.
\end{abstract}

\section{Introduction}

As an important part of the meson family, the kaon subfamily has become more and more abundant with experimental progress on the observations of the kaons in the past decades. Until now, the Particle Data Group (PDG) has collected dozens of the kaons [1]. When facing so abundantly many kaons, it is one of the main tasks of the present study of light hadron spectroscopy to categorize them into the family and another task is to investigate the higher radial and orbital excitations.

Before the present work, there were some theoretical papers related to kaons. For example, 30 years ago, Godfrey and Isgur [2] developed a relativistic quark model, the socalled Godfrey-Isgur (GI) model, by which they studied the mass spectrum of hadrons including kaons. In 2002, Barnes et al. [3] further investigated the strong decays of the observed kaons, which have masses less than $2.2 \mathrm{GeV}$, where the ${ }^{3} P_{0}$

\footnotetext{
a e-mail: xiangliu@lzu.edu.cn

be-mail: matsuki@tokyo-kasei.ac.jp
}

quark model associated with a simple harmonic oscillator (SHO) wave function was adopted in their calculation. In 2009, Ebert et al. [4] analyzed the mass spectrum and Regge trajectories of the kaons by their relativistic quark model.

Due to the present experimental progress on kaons, it is a suitable time to systematically carry out phenomenological study of the kaons. In this work, we first calculate the mass spectra of the kaon family by applying the modified GI model $[5,6]$, where the screening effect is taken into account. Fitting some well-established kaon states, we fix the parameters in the model, which are adopted when calculating the masses of other kaon states. Comparing theoretical results with experimental data, we obtain the structure information of the discussed kaons. Especially, we predict some radial ground states of the kaon which are still missing in experiments, e.g. $K_{4}(2310)\left(1^{1} G_{4}\right)$. Using our potential model approach, the spatial wave functions of the kaons studied can be numerically calculated, which we take as input when studying their Okubo-Zweig-Iizuka (OZI)-allowed two-body strong decays. For further testing the properties of the kaons, we study their OZI-allowed two-body strong decays, which provide valuable information of their partial and total decay widths, where one uses the quark pair creation (QPC) model which was proposed in Ref. [7] and extensively applied to studies of other hadrons in Refs. [8-34]. Analyzing mass spectra and calculating strong decay behaviors, we finally identify their $n^{2 S+1} L_{J}$ quantum numbers, which reflect the inner structure of the kaons under discussion. Here, we predict the strong decay behaviors of some kaon states, e.g. $K_{4}(2310)\left(1^{1} G_{4}\right)$ has a wide width about $710-880 \mathrm{MeV}$, and mainly decays into $K_{4}^{*}(2045) \pi, K_{3}^{*}(1780) \pi, K \rho_{3}(1690)$ and $K a_{2}$. The study presented in this work is helpful for establishing the kaon family by including higher radial and orbital excitations. 
This paper is organized as follows. After Introduction, in Sect. 2 we explain the modified Godfrey-Isgur model and the QPC model. In Sect. 3, we adopt the modified Godfrey-Isgur model by including the screening effect to study the mass spectra of the kaon family. Making a comparison between theoretical and experimental results, we further obtain the structure information of the observed kaons. In Sect. 4, we present the detailed study of the OZI-allowed two-body strong decays of the discussed kaons. The paper ends with conclusions and discussion.

\section{Phenomenological quark models adopted in this work}

In our calculation, two phenomenological quark models are adopted, i.e., the modified GI model with the color screening effect $^{1}$ and the QPC model. The modified GI quark model is applied to calculate the mass spectrum of the kaon family, by which we obtain the structure information of the observed kaon candidates. Then we further test the possible assignments by comparing the theoretical results of their two-body OZI-allowed decays with the experimental data, where the QPC model is used to calculate their strong decays.

In the following, we will introduce these two models.

\subsection{The modified GI model}

First, we introduce the Godfrey-Isgur (GI) relativized quark model and discuss how the GI model is modified by including the color screening effect. Below we describe the detailed procedure and equations actually done by us because those are necessary in our work but are not familiar to the general reader. Some are common to Godfrey and Isgur.

The interaction between quark and antiquark in the GI model [2] is described by the Hamiltonian

$\tilde{H}=\left(p^{2}+m_{u / d}^{2}\right)^{1 / 2}+\left(p^{2}+m_{s}^{2}\right)^{1 / 2}+\tilde{V}_{\mathrm{eff}}(\mathbf{p}, \mathbf{r})$,

where $m_{u / d}$ and $m_{s}$ are the masses of $u / d$ and $s$ quarks, respectively, i.e., $m_{u}=m_{d}=220 \mathrm{MeV}, m_{s}=419 \mathrm{MeV}$. $\tilde{V}_{\text {eff }}(\mathbf{p}, \mathbf{r})=\tilde{H}^{\text {conf }}+\tilde{H}^{\text {hyp }}+\tilde{H}^{\text {SO }}$ is the effective potential of the $q \bar{q}$ interaction which can be obtained from on-shell $q \bar{q}$ scattering amplitudes in the center-of-mass (CM) frame [2] and relativistic effect corrections. The quantities with tilde will be defined later. On the other hand $\tilde{V}_{\text {eff }}(\mathbf{p}, \mathbf{r})$ also consists

\footnotetext{
${ }^{1}$ When studying the mass spectrum of the mesons, there are approaches like the Dyson-Schwinger and Bethe-Salpeter equations, which are directly related to QCD. However, such theory-based or theory-linked approaches still have some limitations in describing higher excitations of the mesons. Instead of theoretical approaches, one may apply phenomenological models to deal with such subjects. Here, the modified GI model is adopted to calculate the mass spectrum of pseudoscalar mesons.
}

of two main parts. The first one is a $\gamma^{\mu} \otimes \gamma_{\mu}$ short-distance interaction of one-gluon exchange and the second part is a $1 \otimes 1$ long-distance color confining linear interaction, which is suggested by the lattice QCD [35-39].

In the nonrelativistic limit, $V_{\text {eff }}(\mathbf{p}, \mathbf{r})$ without tilde is transformed into the familiar nonrelativistic potential $V_{\text {eff }}(r)$ $[2,40]$

$V_{\text {eff }}(r)=H^{\text {conf }}+H^{\text {hyp }}+H^{\text {so }}$

with

$$
\begin{aligned}
H^{\mathrm{conf}}= & {\left[-\frac{3}{4}(c+b r)+\frac{\alpha_{s}(r)}{r}\right]\left(\boldsymbol{F}_{1} \cdot \boldsymbol{F}_{2}\right) } \\
= & S(r)+G(r) \\
H^{\text {hyp }}= & -\frac{\alpha_{s}(r)}{m_{u / d} m_{s}}\left[\frac{8 \pi}{3} \boldsymbol{S}_{1} \cdot \boldsymbol{S}_{2} \delta^{3}(\boldsymbol{r})+\frac{1}{r^{3}}\left(\frac{3 \boldsymbol{S}_{1} \cdot \boldsymbol{r} \boldsymbol{S}_{2} \cdot \boldsymbol{r}}{r^{2}}\right.\right. \\
& \left.\left.-\boldsymbol{S}_{1} \cdot \boldsymbol{S}_{2}\right)\right]\left(\boldsymbol{F}_{1} \cdot \boldsymbol{F}_{2}\right) \\
H^{\text {so }}= & H^{\text {so(cm) }}+H^{\text {so(tp) }},
\end{aligned}
$$

where $H^{\text {conf }}$ is the spin-independent potential which contains a linear confining potential $S(r)=b r+c$ and the one-gluon exchange potential $G(r)=-4 \alpha_{s}(r) / 3 r, H^{\text {hyp }}$ and $H^{\text {SO }}$ are the color-hyperfine interaction and the spin-orbit interaction, respectively. It can be noted that $\boldsymbol{F}_{1}\left(\boldsymbol{F}_{2}\right)=\lambda_{1}\left(-\lambda_{2}^{*}\right) / 2$, where $\lambda_{i}$ is the Gell-Mann matrix. For the meson, $\left(\boldsymbol{F}_{1} \cdot \boldsymbol{F}_{2}\right)=$ $-4 / 3$. Additionally, the subscripts 1 and 2 denote quark and antiquark, respectively.

In Eqs. (3) and (4), the running coupling constant $\alpha_{s}(r)$ has the following form:

$\alpha_{s}(r)=\sum_{k} \frac{2 \alpha_{k}}{\sqrt{\pi}} \int_{0}^{\gamma_{k} r} e^{-x^{2}} \mathrm{~d} x$,

where $k$ is from 1 to 3 and corresponding $\alpha_{k}$ and $\gamma_{k}$ are constant, $\alpha_{1,2,3}=0.25,0.15,0.2$ and $\gamma_{1,2,3}=\frac{1}{2}, \frac{\sqrt{10}}{2}, \frac{\sqrt{1000}}{2}$ [2]. For the color-hyperfine interaction $H^{\text {hyp }}$, the first term stands for contact interaction and second term is a typical form of tensor interaction, here $S_{1}\left(S_{2}\right)$ denotes the spin of the quark (antiquark).

In Eq. (5), the spin-orbit interaction can be divided into two types in which $H^{\text {so(cm) }}$ is the color-magnetic term and $H^{\text {so(tp) }}$ is the Thomas-precession term. Their expression can be written as

$$
\begin{aligned}
H^{\mathrm{so}(\mathrm{cm})}= & -\frac{\alpha_{s}(r)}{r^{3}}\left(\frac{1}{m_{u / d}}+\frac{1}{m_{s}}\right) \\
& \times\left(\frac{\boldsymbol{S}_{1}}{m_{u / d}}+\frac{\boldsymbol{S}_{2}}{m_{s}}\right) \cdot \boldsymbol{L}\left(\boldsymbol{F}_{1} \cdot \boldsymbol{F}_{2}\right), \\
H^{\mathrm{so}(\mathrm{tp})}= & -\frac{1}{2 r} \frac{\partial H^{\mathrm{conf}}}{\partial r}\left(\frac{\boldsymbol{S}_{1}}{m_{u / d}^{2}}+\frac{\boldsymbol{S}_{2}}{m_{s}^{2}}\right) \cdot \boldsymbol{L},
\end{aligned}
$$


where $\boldsymbol{L}$ is the orbital momentum between quark and antiquark.

Noting that the above interaction potentials are obtained in the nonrelativistic limit, and they can optimized by introducing the phenomenological relativistic effects. In the GI model, the relativistic effects are imposed into the model mainly by two ways. Firstly, a smearing function $\rho\left(\boldsymbol{r}-\boldsymbol{r}^{\prime}\right)$ is introduced to incorporate the effects of an internal motion inside a meson and nonlocality of interactions between quark and antiquark. A smearing transformation is given by

$\tilde{f}(r)=\int d^{3} r^{\prime} \rho\left(r-r^{\prime}\right) f\left(r^{\prime}\right)$,

with

$\rho\left(r-r^{\prime}\right)=\frac{\sigma^{3}}{\pi^{3 / 2}} e^{-\sigma^{2}}\left(r-r^{\prime}\right)^{2}$,

$\sigma=\sqrt{s^{2}\left(\frac{2 m_{u / d} m_{s}}{m_{u / d}+m_{s}}\right)^{2}+\sigma_{0}^{2}\left(\frac{1}{2}\left(\frac{4 m_{u / d} m_{s}}{\left(m_{u / d}+m_{s}\right)^{2}}\right)^{4}+\frac{1}{2}\right)}$,

where $\sigma_{0}=1.80 \mathrm{GeV}$ and $s=1.55$, are the universal parameters in the GI model, $f(r)$ is a arbitrary function and notation tilde stands for that the expression has been performed smearing transformation. By the smearing transformation, the one-gluon exchange potential $G(r)=-4 \alpha_{s}(r) /(3 r)$ and linear confined potential $S(r)=b r+c$ are changed; thus

$$
\begin{aligned}
\tilde{G}(r)= & -\sum_{k} \frac{8 \alpha_{k}}{3 \sqrt{\pi} r} \int_{0}^{\tau_{k} r} e^{-x^{2}} \mathrm{~d} x, \\
\tilde{S}(r)=b r & {\left[\frac{e^{-\sigma^{2} r^{2}}}{\sqrt{\pi} \sigma r}\right.} \\
+ & \left.\left(1+\frac{1}{2 \sigma^{2} r^{2}}\right) \frac{2}{\sqrt{\pi}} \int_{0}^{\sigma r} e^{-x^{2}} \mathrm{~d} x\right]+c,
\end{aligned}
$$

where

$\tau_{k}=\frac{1}{\sqrt{\frac{1}{\sigma^{2}}+\frac{1}{\gamma_{k}^{2}}}}$.

Secondly, a general expression of the potential should be dependent on the CM momentum of the interacting quarks. So the momentum-dependent effect is achieved by introducing momentum-dependent factors which will go to unity in the nonrelativistic limit. In a semiquantitative relativistic treatment, the smeared one-gluon exchange potential term $\tilde{G}(r)$ and the smeared hyperfine interactions (or spinorbit interaction) $\tilde{V}^{i}$ should be modified with the following momentum-dependent factors:

$$
G^{\prime}(r)=\left(1+\frac{p^{2}}{E_{1} E_{2}}\right)^{1 / 2} \tilde{G}(r)\left(1+\frac{p^{2}}{E_{1} E_{2}}\right)^{1 / 2},
$$

$$
\begin{aligned}
\tilde{G}_{i j}^{s o(v)} & =\left(\frac{m_{i} m_{j}}{E_{i} E_{j}}\right)^{1 / 2+\epsilon_{s o(v)}} \tilde{G}(r)\left(\frac{m_{i} m_{j}}{E_{i} E_{j}}\right)^{1 / 2+\epsilon_{s o(v)}}, \\
\tilde{G}_{12}^{c} & =\left(\frac{m_{1} m_{2}}{E_{1} E_{2}}\right)^{1 / 2+\epsilon_{c}} \tilde{G}(r)\left(\frac{m_{1} m_{2}}{E_{1} E_{2}}\right)^{1 / 2+\epsilon_{c}}, \\
\tilde{G}_{12}^{t} & =\left(\frac{m_{1} m_{2}}{E_{1} E_{2}}\right)^{1 / 2+\epsilon_{t}} \tilde{G}(r)\left(\frac{m_{1} m_{2}}{E_{1} E_{2}}\right)^{1 / 2+\epsilon_{t}}, \\
\tilde{S}_{11}^{s o(s)} & =\left(\frac{m_{1}^{2}}{E_{1}^{2}}\right)^{1 / 2+\epsilon_{s o(s)}} \tilde{S}(r)\left(\frac{m_{1}^{2}}{E_{1}^{2}}\right)^{1 / 2+\epsilon_{s o(s)}}, \\
\tilde{S}_{22}^{s o(s)} & =\left(\frac{m_{2}^{2}}{E_{2}^{2}}\right)^{1 / 2+\epsilon_{s o(s)}} \tilde{S}(r)\left(\frac{m_{2}^{2}}{E_{2}^{2}}\right)^{1 / 2+\epsilon_{s o(s)}},
\end{aligned}
$$

where $E_{1}=\sqrt{m_{u / d}^{2}+p^{2}}$ and $E_{2}=\sqrt{m_{s}^{2}+p^{2}}$ are the energies of the quark and antiquark in the meson, and $m_{1}=m_{u / d}$, $m_{2}=m_{s}, \epsilon_{i}$ is parameter for a different type of hyperfine and spin-orbit interactions, which include the contact, tensor, vector spin-orbit and scalar spin-orbit potentials. Here, vector spin-orbit and scalar spin-orbit potentials correspond to Eq. (5) related to one-gluon exchange and confinement term, respectively. So the total Hamiltonian can be written as

$$
\begin{aligned}
\hat{H}= & \left(p^{2}+m_{u / d}^{2}\right)^{1 / 2}+\left(p^{2}+m_{s}^{2}\right)^{1 / 2} \\
& +\tilde{H}^{\text {conf }}+\tilde{H}^{\text {hyp }}+\tilde{H}^{s o}
\end{aligned}
$$

with

$$
\begin{aligned}
\tilde{H}_{12}^{c o n f} & =G^{\prime}(r)+\tilde{S}(r), \\
\tilde{H}^{s o} & =\tilde{H}^{s o(v)}+\tilde{H}^{s o(s)},
\end{aligned}
$$

where

$$
\begin{aligned}
\tilde{H}^{s o(v)}= & \frac{\mathbf{S}_{1} \cdot \mathbf{L}}{2 m_{u / d}^{2} r} \frac{\partial \tilde{G}_{11}^{s o(v)}}{\partial r}+\frac{\mathbf{S}_{2} \cdot \mathbf{L}}{2 m_{s}^{2} r} \frac{\partial \tilde{G}_{22}^{s o(v)}}{\partial r} \\
& +\frac{\left(\mathbf{S}_{1}+\mathbf{S}_{2}\right) \cdot \mathbf{L}}{m_{u / d} m_{s}} \frac{1}{r} \frac{\partial \tilde{G}_{12}^{s o(v)}}{\partial r}, \\
\tilde{H}^{s o(s)}= & -\frac{\mathbf{S}_{1} \cdot \mathbf{L}}{2 m_{u / d}^{2} r} \frac{\partial \tilde{S}_{11}^{s o(s)}}{\partial r}-\frac{\mathbf{S}_{2} \cdot \mathbf{L}}{2 m_{s}^{2} r} \frac{\partial \tilde{S}_{22}^{s o(s)}}{\partial r}, \\
\tilde{H}_{12}^{\text {hyp }}= & \tilde{H}_{12}^{\text {tensor }}+\tilde{H}_{12}^{c},
\end{aligned}
$$

where

$$
\begin{aligned}
\tilde{H}_{12}^{\text {tensor }}= & -\left(\frac{\mathbf{S}_{1} \cdot \mathbf{r} \mathbf{S}_{2} \cdot \mathbf{r} / r^{2}-\frac{1}{3} \mathbf{S}_{1} \cdot \mathbf{S}_{2}}{m_{u / d} m_{s}}\right) \\
& \times\left(\frac{\partial^{2}}{\partial r^{2}}-\frac{1}{r} \frac{\partial}{\partial r}\right) \tilde{G}_{12}^{t}, \\
\tilde{H}_{12}^{c}= & \frac{2 \mathbf{S}_{1} \cdot \mathbf{S}_{2}}{3 m_{u / d} m_{s}} \nabla^{2} \tilde{G}_{12}^{c} .
\end{aligned}
$$

For solving the Schrödinger equation, $\hat{H} \Psi=E \Psi$, with $\hat{H}$ shown in Eq. (21), the simple harmonic oscillators (SHO) 
wave function will be employed. In the configuration space, the SHO wave function has the form

$\Psi_{n L M_{L}}(\mathbf{r})=R_{n L}(r, \beta) Y_{L M_{L}}\left(\Omega_{r}\right)$,

$\Psi_{n L M_{L}}(\mathbf{p})=R_{n L}(p, \beta) Y_{L M_{L}}\left(\Omega_{p}\right)$,

with

$$
\begin{aligned}
R_{n L}(r, \beta)= & \beta^{3 / 2} \sqrt{\frac{2 n !}{\Gamma(n+L+3 / 2)}}(\beta r)^{L} e^{\frac{-r^{2} \beta^{2}}{2}} \\
& \times L_{n}^{L+1 / 2}\left(\beta^{2} r^{2}\right), \\
R_{n L}(p, \beta)= & \frac{(-1)^{n}(-i)^{L}}{\beta^{3 / 2} e^{-\frac{p^{2}}{2 \beta^{2}}}} \\
& \times \sqrt{\frac{2 n !}{\Gamma(n+L+3 / 2)}}\left(\frac{p}{\beta}\right)^{L} L_{n}^{L+1 / 2}\left(\frac{p^{2}}{\beta^{2}}\right),
\end{aligned}
$$

where $Y_{L M_{L}}(\Omega)$ is a spherical harmonic function with orbital angular momentum quantum number $\mathrm{L}$, and $L_{n-1}^{L+1 / 2}(x)$ is an associated Laguerre polynomial, and $\beta$ is a parameter of oscillator radial wave function. A series of SHO wave functions with different radial quantum numbers $n$ can be regarded as a complete basis to expand the exact radial wave function of the meson state, in this case, the meson mass spectrum can be obtained by diagonalizing the Hamiltonian matrix of Eq. (21) by the above SHO basis. The total wave function of the meson is composed of color, flavor, spin, space wave function, and the spin wave functions $\chi$ are

$$
\begin{aligned}
\chi_{00} & =\frac{1}{\sqrt{2}}(\uparrow \downarrow-\downarrow \uparrow), \\
\chi_{11} & =\uparrow \uparrow, \\
\chi_{10} & =\frac{1}{\sqrt{2}}(\uparrow \downarrow+\downarrow \uparrow), \\
\chi_{1-1} & =\downarrow \downarrow .
\end{aligned}
$$

The space-spin wave function $R_{n L}(r, \beta) \phi_{L S J M}$ with total angular quantum number $J$ can be constructed by coupling $L \otimes S$ and has the form

$\phi_{L S J M}=\sum_{M_{L} M_{S}} C\left(L M_{L} S M_{S} ; J M\right) Y_{L M_{L}}\left(\Omega_{r}\right) \chi_{S M_{S}}$,

where $C\left(L M_{L} S M_{S} ; J M\right)$ is the Clebsch-Gordan coefficient. For the matrix element $\langle\alpha|\hat{V}(r, \hat{p})| \beta\rangle$ where $|\alpha\rangle$ and $|\beta\rangle$ are arbitrary SHO basis with quantum number $\{n, J, L, S\}$ and $\left\{n^{\prime}, J^{\prime}, L^{\prime}, S^{\prime}\right\}$. It is noted that the color and flavor wave function of the meson have no contributions for the matrix element of Hamiltonian, and we have the general expression

$$
\begin{aligned}
\langle\alpha|\hat{V}(r, \hat{p})| \beta\rangle & =\langle\alpha|f(p) g(r)| \beta\rangle \\
& =\sum_{n}\langle\alpha|f(p)| n\rangle\langle n|g(r)| \beta\rangle .
\end{aligned}
$$

After calculating each matrix element, the mass and wave function of meson could be obtained and they also are available for the following strong decay process.

Although the GI model has achieved great success in describing the meson spectrum, there still exists a discrepancy between the predictions given by the GI model and recent experimental observations. The previous work [5] presented a modified GI model with a screening potential whose predictions can be well consistent with the experimental data for the charm-strange mesons. For higher excitation states, the authors of Ref. [5] believe that a screening effect plays a very important role which could be introduced by the transformation $b r+c \rightarrow \frac{b\left(1-e^{-\mu r}\right)}{\mu}+c$, where $\mu$ is a screening parameter whose particular value is need to be fixed by the comparisons between theory and experiment. For a modified confinement potential one also needs to make a similar relativistic correction to the one mentioned in the GI model. Then we further write $V^{\text {scr }}(r)$ in the way given in Eq. (13),

$\tilde{V}^{\mathrm{scr}}(r)=\int d^{3} \boldsymbol{r}^{\prime} \rho\left(\boldsymbol{r}-\boldsymbol{r}^{\prime}\right) \frac{b\left(1-e^{-\mu r^{\prime}}\right)}{\mu}$.

By inserting the form of $\rho\left(\boldsymbol{r}-\boldsymbol{r}^{\prime}\right)$ in Eq. (11) into the above expression and finishing this integration, the concrete expression for $\tilde{V}^{\text {scr }}(r)$ is given by

$$
\begin{aligned}
\tilde{V}^{\mathrm{scr}}(r)= & \frac{b}{\mu r}\left[r+e^{\frac{\mu^{2}}{4 \sigma^{2}}+\mu r} \frac{\mu+2 r \sigma^{2}}{2 \sigma^{2}}\right. \\
& \times\left(\frac{1}{\sqrt{\pi}} \int_{0}^{\frac{\mu+2 r \sigma^{2}}{2 \sigma}} e^{-x^{2}} \mathrm{~d} x-\frac{1}{2}\right) \\
& -e^{\frac{\mu^{2}}{4 \sigma^{2}}-\mu r} \frac{\mu-2 r \sigma^{2}}{2 \sigma^{2}} \\
& \left.\times\left(\frac{1}{\sqrt{\pi}} \int_{0}^{\frac{\mu-2 r \sigma^{2}}{2 \sigma}} e^{-x^{2}} \mathrm{~d} x-\frac{1}{2}\right)\right] .
\end{aligned}
$$

It is worth mentioning that after the confinement potential is replaced with a screening potential, other treatments are similar to the original GI model including the calculation of matrix elements of the Hamiltonian.

\subsection{The QPC model}

The QPC model was first proposed by Micu [7] and further developed by the Orsay group [8,41-44]. This model was widely applied to the OZI-allowed two-body strong decay of hadrons in Refs. $[9,10,13,15,17,19-24,27-30,32,33,45-$ 49]. 
For a decay process $A \rightarrow B+C$, we can write

$\langle B C|\mathcal{T}| A\rangle=\delta^{3}\left(\mathbf{P}_{B}+\mathbf{P}_{C}\right) \mathcal{M}^{M_{J_{A}} M_{J_{B}} M_{J_{C}},}$

where $\mathbf{P}_{B(C)}$ is a three-momentum of a meson $B(C)$ in the rest frame of a meson $A$. A superscript $M_{J_{i}}(i=A, B, C)$ denotes an orbital magnetic momentum. The transition operator $\mathcal{T}$ is introduced to describe a quark-antiquark pair creation from vacuum, which has the quantum number $J^{P C}=$ $0^{++}$, i.e., $\mathcal{T}$ can be expressed as

$$
\begin{aligned}
\mathcal{T}= & -3 \gamma \sum_{m}\langle 1 m ; 1-m \mid 00\rangle \int \mathrm{d} \mathbf{p}_{3} \mathrm{~d} \mathbf{p}_{4} \delta^{3}\left(\mathbf{p}_{3}+\mathbf{p}_{4}\right) \\
& \times \mathcal{Y}_{1 m}\left(\frac{\mathbf{p}_{3}-\mathbf{p}_{4}}{2}\right) \\
& \times \chi_{1,-m}^{34} \phi_{0}^{34}\left(\omega_{0}^{34}\right)_{i j} b_{3 i}^{\dagger}\left(\mathbf{p}_{3}\right) \mathrm{d}_{4 j}^{\dagger}\left(\mathbf{p}_{4}\right),
\end{aligned}
$$

which is constructed in a completely phenomenological way to reflect the creation of a quark-antiquark pair from vacuum, where the quark and antiquark are denoted by indices 3 and 4, respectively. A dimensionless parameter $\gamma$ depicts the strength of the creation of $q \bar{q}$ from vacuum; the concrete values of the parameter $R$ will be discussed below. We have $\mathcal{Y}_{\ell m}(\mathbf{p})=|\mathbf{p}|^{\ell} Y_{\ell m}(\mathbf{p})$, the solid harmonics. $\chi, \phi$, and $\omega$ denote the spin, flavor, and color wave functions respectively, which can be treated separately. Indices $i$ and $j$ denote the color of a $q \bar{q}$ pair.

By the Jacob-Wick formula [50], the decay amplitude is expressed as

$$
\begin{aligned}
\mathcal{M}^{J L}(\mathbf{P})= & \frac{\sqrt{4 \pi(2 L+1)}}{2 J_{A}+1} \sum_{M_{J_{B}} M_{J_{C}}}\left\langle L 0 ; J M_{J_{A}} \mid J_{A} M_{J_{A}}\right\rangle \\
& \times\left\langle J_{B} M_{J_{B}} ; J_{C} M_{J_{C}} \mid J_{A} M_{J_{A}}\right\rangle \mathcal{M}^{M_{J_{A}} M_{J_{B}} M_{J_{C}}},
\end{aligned}
$$

and the general decay width reads

$\Gamma=\frac{\pi}{4} \frac{|\mathbf{P}|}{m_{A}^{2}} \sum_{J, L}\left|\mathcal{M}^{J L}(\mathbf{P})\right|^{2}$,

where $m_{A}$ is the mass of an initial state $A$. In our calculation, we need the spatial wave functions of the discussed kaons and iso-scalar and iso-vector light mesons. These can be numerically obtained by the modified GI model.

\section{Mass spectrum analysis}

Although the GI model has succeeded in describing the ground states of the kaon family, it does not well describe the excited states. Since unquenched effects are important for a heavy-light system, it is better to adopt the modified GI model (MGI) [5,6] which uses a screening potential with a new parameter $\mu$. The parameter $\mu$ describes the inverse
Table 1 Parameters and their values in this work and GI models

\begin{tabular}{lll}
\hline Parameter & This work & GI [2] \\
\hline$b$ & 0.2555 & 0.18 \\
$c$ & -0.3492 & -0.253 \\
$\mu$ & 0.1 & 0 \\
$\epsilon_{\text {sov }}$ & -0.01700 & -0.035 \\
$\epsilon_{c}$ & -0.1396 & -0.168 \\
$\epsilon_{t}$ & 0.03600 & 0.025 \\
$\epsilon_{\text {sos }}$ & 0.06772 & 0.055 \\
\hline
\end{tabular}

of the size of screening. To use the MGI model to calculate the kaon family spectra, it is better to determine the value of a new parameter $\mu$ considering two features: The first is when we use the same parameter set as in Ref. [2] and add a new parameter $\mu$, the mass of the ground state of the kaon family will be lower than the experiments. The second one is that the value of $\mu$ may be not so small like as one in Refs. $[5,6]$. In fact, in bottomonium and charmonium states, Refs. $[51,52]$ give a $\mu$ value of about $0.1 \mathrm{GeV}$, which is larger than the one in Refs. [5,6]. Since we do not know the real value of $\mu$ in the kaon family beforehand, we need to adjust the parameters by fitting with the experiments data. At first, the quark masses should be the same for all meson families. Secondly, we do not adjust the values of $\Lambda$ and $\alpha_{s}$ for the same reason. Since $\sigma_{0}$ and $s$ are universal parameters which are resolved by the $Q \bar{Q}$ system, we do not vary them in our fit. The confining term $b r+c$ will be replaced by the screening potential, so their parameters should be fitted again. The relativistic effects should be adapted to a different system with the different quark masses. So we fix the following seven parameters listed in Table 1 by fitting 11 experimental data as listed in Table 2.

In Table 2, we select 11 experimental data of kaons listed in PDG and optimize these kaon masses to determine seven parameters in Table 1. This optimization has $\chi^{2} / n=12.6$, which is smaller than 90.2 for the GI model as shown in Table 2. Another reason why we choose these kaons to fix the parameters in our model is that there does not exist a mixture between $n^{1} L_{L}$ and $n^{3} L_{L}$ states for these kaons. In order to obtain the optimum values of parameters and global and good fit of 11 data, we set "Error in fitting" in Table 2 so that the first two experimental data, corresponding to $K$ and $K^{*}$, have artificial larger error values instead of the real errors in the brackets in the fourth column. The results listed in Table 2 show that the MGI model is better than the GI model since the value of $\chi^{2} / n$ of the MGI model is about 7 times smaller than that of the GI model and hence it is safely applied to describing the masses of the selected 11 kaons.

Although the MGI model is better than the GI model to depict 11 experimental data, we need to point out that there may exist a $\sim \mathcal{O}(100 \mathrm{MeV})$ deviation between experimen- 
Table 2 The experimental data [1] fitted in our work. $\chi^{2}=((\mathrm{Th}-\text { Exp }) / \text { Error })^{2}$, where Th, Exp, and Error represent the theoretical, experimental results, and experimental error, respectively, and $n$ is the number of the experimental data. We select some established kaon states in PDG [1] for our fitting. The unit of the mass is $\mathrm{MeV}$

\begin{tabular}{llllll}
\hline States & $n^{2 S+1} L_{J}$ & This work & GI [2] & Experiment [1] & Error in fitting \\
\hline$K$ & $1^{1} S_{0}$ & 497.7 & 461.5 & $497.6 \pm 0.013$ & 1.3 \\
$K^{*}(892)$ & $1^{3} S_{1}$ & 896 & 902.8 & $895.8 \pm 0.19$ & 1.9 \\
$K_{0}^{*}(1430)$ & $1^{3} P_{0}$ & 1257 & 1234 & $1425 \pm 50$ & 50 \\
$K_{2}^{*}(1430)$ & $1^{3} P_{2}$ & 1431 & 1428 & $1432.4 \pm 1.3$ & 1.3 \\
$K^{*}(1680)$ & $1^{3} D_{1}$ & 1766 & 1776 & $1717 \pm 27$ & 27 \\
$K_{3}^{*}(1780)$ & $1^{3} D_{3}$ & 1781 & 1794 & $1776 \pm 7$ & 7 \\
$K_{4}^{*}(2045)$ & $1^{3} F_{4}$ & 2058 & 2108 & $2045 \pm 9$ & 9 \\
$K_{5}^{*}(2380)$ & $1^{3} G_{5}$ & 2286 & 2388 & $2382 \pm 14 \pm 19$ & 24 \\
$K_{(1460)}$ & $2^{1} S_{0}$ & 1457 & 1454 & 1460 & 20 \\
$K^{*}(1410)$ & $2^{3} S_{1}$ & 1548 & 1579 & $1414 \pm 15$ & 15 \\
$K_{0}^{*}(1950)$ & $2^{3} P_{0}$ & 1829 & 1890 & $1945 \pm 10 \pm 20$ & 22 \\
$\chi^{2} / n$ & & 12.6 & 90.2 & & \\
\hline
\end{tabular}

tal and fitting results for several kaons, which is shown in Table 2. Such a difference of experimental and theoretical results may be due to the precision of the experiment. For example, there is only one experiment [1] for $K_{5}^{*}(2380)$ and $K_{0}^{*}(1950)$. The confirmation of $K_{5}^{*}(2380)$ and $K_{0}^{*}(1950)$ is still absent. Thus, further experimental measurement of the resonance parameters of these kaons will be helpful to clarify this difference of experimental and theoretical results.

By using the parameters shown in Table 1 as input, we further calculate the masses of other kaons, which are collected in Table 3, where we do not consider the mixing of states with $n^{1} L_{L}$ and $n^{3} L_{L}$. Usually, there exists a mixture of the $n^{1} L_{L}$ and $n^{3} L_{L}$ states, i.e. [53]

$$
\left(\begin{array}{c}
|n L\rangle \\
\left|n L^{\prime}\right\rangle
\end{array}\right) \approx\left(\begin{array}{cc}
\cos \theta_{n L} & \sin \theta_{n L} \\
-\sin \theta_{n L} & \cos \theta_{n L}
\end{array}\right)\left(\begin{array}{l}
\left|n^{1} L_{L}\right\rangle \\
\left|n^{3} L_{L}\right\rangle
\end{array}\right)
$$

where $|n L\rangle$ and $\left|n L^{\prime}\right\rangle$ are two mixing physical states and $\theta_{n L}$ is the corresponding mixing angle. Introducing such mixing states, we find two mass relations $m(n L)<m\left(n^{1} L_{L}\right)$ and $m\left(n L^{\prime}\right)>m\left(n^{3} L_{L}\right)$, which can be applied to identify these observed kaons with the same $J^{P}$ quantum number. Thus, we need to combine the mass relations and mass spectrum of the kaons listed in Table 3 with the experimental data to further shed light on the properties of other observed kaons. We conclude that

1. Both $K_{1}$ (1270) with $M=(1272 \pm 7) \mathrm{MeV}$ [1] and $K_{1}(1400)$ with mass $M=(1403 \pm 7) \mathrm{MeV}$ [1] have $J^{P}=1^{+}$quantum numbers. $K_{1}(1270)$ and $K_{1}(1400)$ are the mixture of $1^{1} P_{1}$ and $1^{3} P_{1}$ states, i.e., $K_{1}(1270)$ and $K_{1}(1400)$ correspond to $1 P$ and $1 P^{\prime}$ states, respectively.

2. $K_{2}(1770)$ has $J^{P}=2^{-}$and $M=(1773 \pm 8) \mathrm{MeV}[1]$, while $K_{2}(1820)$ has $J^{P}=2^{-}$and $M=(1816 \pm 13)$ $\mathrm{MeV}$ [1]. $K_{2}(1770)$ and $K_{2}(1820)$, which correspond to the $1 D$ and $1 D^{\prime}$ states, respectively, are the mixture of the $1^{1} D_{2}$ and $1^{3} D_{2}$ states.

3. $K_{1}(1650)$ has $J^{P}=1^{+}$and $M=(1650 \pm 50) \mathrm{MeV}$ [1]. Since the mass of $K_{1}(1650)$ is smaller than that of the $2^{1} P_{1}$ state obtained in Table 3 , thus we suggest that $K_{1}(1650)$ can be assigned as a $2 P$ state. There must exist a partner, the $2 P^{\prime}$ state, which is still missing in experiments.

4. We suggest that $K(1830)$ is a $3^{1} S_{0}$ state. Later, we will test this assignment by studying its decay behavior.

5. $K_{2}^{*}(1980)$ with $J^{P}=2^{+}$and $M=(2020 \pm 20) \mathrm{MeV}$ [54] is either a $2^{3} P_{2}$ state or a $1^{3} F_{2}$ state.

6. $K_{2}(2250)$ with $J^{P}=2^{-}$and $M=(2247 \pm 17) \mathrm{MeV}$ [1] is the candidate of $2 D^{\prime}$, which is the mixture of the $2^{1} D_{2}$ and $2^{3} D_{2}$ states.

7. $K_{3}(2320)$ has $J^{P}=3^{+}$and $M=(2324 \pm 24) \mathrm{MeV}[1]$. The possible assignment of $K_{3}(2320)$ is the $2 F$ state, which is the mixture of states $K\left(2^{1} F_{3}\right)$ and $K\left(2^{3} F_{3}\right)$. As the partner of $K_{3}(2320), K\left(2 F^{\prime}\right)$ is till absent in experiments. In addition, we should mention that $1 F$ and $1 F^{\prime}$ in the kaon family are still missing.

8. $K_{4}(2500)$ with $J^{P}=4^{-}$and $M=(2490 \pm 20) \mathrm{MeV}$ [1] can be a $2 G$ state, while its partner $K\left(2 G^{\prime}\right)$ and two kaons $K(1 G)$ and $K\left(1 G^{\prime}\right)$ are still missing in experiments.

Surely, the above conclusions of possible quantum states are only from the point of mass spectra view. If we want to clearly study particle properties further, we also need to investigate the decay behaviors, especially strong decays, and a detailed study will be given in the next section.

\section{OZI-allowed two-body strong decays}

In the previous section, calculating the spectra of the kaon family, we obtain kaon wave functions, too, at the same time, 
Table 3 The masses of other kaons obtained by the MGI model and comparison with those from other potential models. The unit of the mass is $\mathrm{MeV}$

\begin{tabular}{|c|c|c|c|c|c|c|}
\hline$n^{2 S+1} L_{J}$ & This work & GI [2] & Ref. [4] & Ref. [3] & $n^{2 S+1} L_{J}$ & This work \\
\hline $1^{1} P_{1}$ & 1364 & 1352 & - & - & $3^{1} D_{2}$ & 2380 \\
\hline $1^{3} P_{1}$ & 1377 & 1366 & - & - & $3^{3} D_{1}$ & 2385 \\
\hline $1^{1} D_{2}$ & 1778 & 1791 & 1709 & - & $3^{3} D_{2}$ & 2388 \\
\hline $1^{3} D_{2}$ & 1789 & 1804 & 1824 & - & $3^{3} D_{3}$ & 2382 \\
\hline $1^{1} F_{3}$ & 2075 & 2131 & 2009 & 2050 & $3^{1} F_{3}$ & 2550 \\
\hline $1^{3} F_{2}$ & 2093 & 2151 & 1964 & 2050 & $3^{3} F_{2}$ & 2560 \\
\hline $1^{3} F_{3}$ & 2084 & 2143 & 2080 & 2050 & $3^{3} F_{3}$ & 2546 \\
\hline $1^{1} G_{4}$ & 2309 & 2422 & 2255 & - & $3^{3} F_{4}$ & 2533 \\
\hline $1^{3} G_{3}$ & 2336 & 2458 & 2207 & - & $3^{1} G_{4}$ & 2673 \\
\hline $1^{3} G_{4}$ & 2317 & 2433 & 2285 & - & $3^{3} G_{3}$ & 2687 \\
\hline $2^{1} P_{1}$ & 1840 & 1897 & 1757 & 1850 & $3^{3} G_{4}$ & 2677 \\
\hline $2^{3} P_{1}$ & 1861 & 1928 & 1893 & 1850 & $3^{3} G_{5}$ & 2662 \\
\hline $2^{3} P_{2}$ & 1870 & 1938 & 1896 & 1850 & $4^{1} S_{0}$ & 2248 \\
\hline $2^{1} D_{2}$ & 2121 & 2238 & 2066 & - & $4^{3} S_{1}$ & 2287 \\
\hline $2^{3} D_{1}$ & 2127 & 2251 & 2063 & - & $4^{1} P_{1}$ & 2422 \\
\hline $2^{3} D_{2}$ & 2131 & 2254 & 2163 & - & $4^{3} P_{0}$ & 2424 \\
\hline $2^{3} D_{3}$ & 2121 & 2237 & 2182 & - & $4^{3} P_{1}$ & 2434 \\
\hline $2^{1} F_{3}$ & 2340 & 2524 & 2348 & - & $4^{3} P_{2}$ & 2438 \\
\hline $2^{3} F_{2}$ & 2356 & 2551 & - & - & $4^{1} D_{2}$ & 2570 \\
\hline $2^{3} F_{3}$ & 2347 & 2536 & - & - & $4^{3} D_{1}$ & 2573 \\
\hline $2^{3} F_{4}$ & 2328 & 2504 & 2436 & - & $4^{3} D_{2}$ & 2575 \\
\hline $2^{1} G_{4}$ & 2520 & 2779 & 2575 & - & $4^{3} D_{3}$ & 2571 \\
\hline $2^{3} G_{3}$ & 2540 & 2814 & - & - & $4^{1} F_{3}$ & 2688 \\
\hline $2^{3} G_{4}$ & 2526 & 2789 & - & - & $4^{3} F_{2}$ & 2695 \\
\hline $2^{3} G_{5}$ & 2504 & 2749 & - & - & $4^{3} F_{3}$ & 2691 \\
\hline $3^{1} S_{0}$ & 1924 & 2065 & - & 1860 & $4^{3} F_{4}$ & 2683 \\
\hline $3^{3} S_{1}$ & 1983 & 2156 & 1950 & - & $4^{1} G_{4}$ & 2782 \\
\hline $3^{1} P_{1}$ & 2177 & 2164 & - & - & $4^{3} G_{3}$ & 2790 \\
\hline $3^{3} P_{0}$ & 2176 & 2160 & - & - & $4^{3} G_{4}$ & 2785 \\
\hline $3^{3} P_{1}$ & 2192 & 2200 & - & - & $4^{3} G_{5}$ & 2776 \\
\hline $3^{3} P_{2}$ & 2198 & 2206 & - & - & & \\
\hline
\end{tabular}

which can be used in the QPC model to study the strong decay of the kaon family. The parameter $\gamma$ in the QPC model is determined by fitting with the experimental data [47]. Thus, there is no free parameter in the QPC model. We obtain $\gamma=$ 10.5 as shown in Table 4.

In the following, we mainly focus on the OZI-allowed two-body strong decay behaviors of these discussed kaons, by which we not only test these possible assignments to the observed kaons, but also provide more abundant predictions of higher radial and orbital excitations in the kaon family.

\section{1 $S$-wave kaons}

Since $K(498)$ and $K^{*}(892)$ were established to be the $1^{1} S_{0}$ and $1^{3} S_{1}$ states in the kaon family, respectively, in this work we do not discuss them, but we present the phenomenological analysis of the $2 S$ and $3 S$ states.

\subsection{1 $2 S$ states}

As the candidate of the $2^{1} S_{0}$ state, $K(1460)$ was listed in PDG. If further checking the experimental data, we find that $K$ (1460) was only reported in Refs. [55,56]. However, in the past 30 years, further experiment as regards $K(1460)$ was missing, which is the reason why $K(1460)$ was removed from the summary table of the PDG.

In Table 5, we give the information on the partial and total decay widths of $K(1460)$ as an $2^{1} S_{0}$ state, in which one can find $K(1460)$ mainly decays into $K^{*} \pi, K \rho$, and $K \omega$. Here, our results are larger than the experimental data for $K^{*} \pi$, 
Table 4 The parameter $\gamma$ fitting in the QPC model. The unit of the width is $\mathrm{MeV}$

\begin{tabular}{lll}
\hline Channels & Experimental data & Numerical result \\
\hline$K^{*} \rightarrow K \pi$ & $50.2 \pm 5$ & 33.5 \\
$K_{0}^{*}(1430) \rightarrow K \pi$ & $267 \pm 36$ & 314 \\
$K_{2}^{*}(1430) \rightarrow K \pi$ & $48.9 \pm 1.7$ & 51.5 \\
$K_{2}^{*}(1430) \rightarrow K^{*} \pi$ & $24.8 \pm 1.7$ & 20.4 \\
$K_{2}^{*}(1430) \rightarrow K \rho$ & $8.7 \pm 0.8$ & 6.13 \\
$K_{2}^{*}(1430) \rightarrow K \omega$ & $2.9 \pm 0.8$ & 1.82 \\
$K_{3}^{*}(1780) \rightarrow K \rho$ & $74 \pm 10$ & 20.1 \\
$K_{3}^{*}(1780) \rightarrow K^{*} \pi$ & $45 \pm 7$ & 28.5 \\
$K_{3}^{*}(1780) \rightarrow K \pi$ & $31.7 \pm 3.7$ & 38.1 \\
$K_{4}^{*}(2045) \rightarrow K \pi$ & $19.6 \pm 3.8$ & 21.0 \\
$K_{4}^{*}(2045) \rightarrow K \phi$ & $2.8 \pm 1.4$ & 3.80 \\
& $\chi^{2}=6.8, \gamma=10.5$ & \\
\hline
\end{tabular}

$K \rho$, and the total width. If we are to establish $K(1460)$ as an $2^{1} S_{0}$ state, we need to clarify these differences between our calculation and the experimental data. We expect an independent experiment to confirm the observation of $K(1460)$. Especially, we suggest precise measurement of the resonance parameters and partial decay widths of $K(1460)$.

In the PDG, $K^{*}(1410)$ is a possible candidate of the $2^{3} S_{1}$ state. However, we must face the following puzzling facts: (1) the mass of $K^{*}(1410)$ is smaller than that of $K(1460)$. Usually, an $2^{3} S_{1}$ state has mass higher than that of an $2{ }^{1} S_{0}$ state. In addition, we also notice the theoretical results of the mass of an $2^{3} S_{1}$ state $[2,4]$ and this work gives the mass of an $2^{3} S_{1}$ state to be $1579,1675,1548$, and $1580 \mathrm{MeV}$, respectively, all of which are larger than the experimental data, $1414 \mathrm{MeV}$, if $K^{*}(1410)$ is an $2^{3} S_{1}$ state. Thus, we need to understand why there exists such a puzzling mass relation for $K^{*}(1410)$ and $K(1460)$. (2) If $K^{*}(1410)$ together with $\rho(1450), \omega(1420)$, and $\phi(1680)$ forms an $2^{3} S_{1}$ nonet, one can notice that the mass of $K^{*}(1410)$ as an $2^{3} S_{1}$ state is a bit small, which was also indicated in Ref. [3].

In Table 5, the obtained partial and total decay widths of $K^{*}(1410)$ as an $2^{3} S_{1}$ are given, where we also compare our result with the experimental data. The main decay modes of $K^{*}(1410)$ include the $K \pi, K^{*} \pi, K \rho$, and $K \eta$ channels. The obtained total decay width of $K^{*}(1410)$ is consistent with the experiment result. We also notice that the ratio $\Gamma_{K \pi} / \Gamma_{\text {Total }}$ obtained in this work is a little bit larger than the experimental value $\left(\Gamma_{K \pi} / \Gamma_{\text {Total }}=(6.6 \pm 1 \pm 0.8) \%\right)$. The above result is gotten by assuming $K^{*}(1410)$ to be an $2^{3} S_{1}$ pure state. In fact, $K^{*}(1410)$ could be a mixture of the $2^{3} S_{1}$ and $1^{3} D_{1}$ states. Thus, in the following, we further discuss such an S-D mixing effect on the ratio $\Gamma_{K \pi} / \Gamma_{\text {Total }}$ of $K^{*}(1410)$. $K^{*}(1410)$ and $K^{*}(1680)$ as a mixture of the $2^{3} S_{1}$ and $1^{3} D_{1}$ states can be expressed as
Table 5 The strong decay widths of the $2 S$ and $3 S$ states. The unit of the width is $\mathrm{MeV}$

\begin{tabular}{|c|c|c|c|}
\hline States & Channels & This work & Experiment \\
\hline \multirow[t]{5}{*}{$\left.K(1460)\left[2^{1} S_{0}\right)\right]$} & $K^{*} \pi$ & 248 & $\sim 109[55]$ \\
\hline & $K \rho$ & 161 & $\sim 34[55]$ \\
\hline & $K \omega$ & 51.2 & - \\
\hline & $K^{*} \eta$ & 8.0 & - \\
\hline & Total & 468 & $\sim 260[55]$ \\
\hline \multirow[t]{7}{*}{$K^{*}(1410)\left[2^{3} S_{1}\right]$} & $K^{*} \pi$ & 81.8 & - \\
\hline & $K \rho$ & 47.4 & - \\
\hline & $K \omega$ & 14.8 & - \\
\hline & $K \pi$ & 34.7 & - \\
\hline & $K \eta$ & 35.4 & - \\
\hline & Total & 214 & $232 \pm 21[1]$ \\
\hline & $\Gamma_{K \pi} / \Gamma_{\text {Total }}$ & $16.2 \%$ & $(6.6 \pm 1 \pm 0.8) \%$ \\
\hline \multirow[t]{11}{*}{$K(1830)\left[3^{1} S_{0}\right]$} & $K^{*}(1410) \pi$ & 105 & - \\
\hline & $K^{*} \pi$ & 34.7 & - \\
\hline & $K_{0}^{*}(1430) \pi$ & 29.8 & - \\
\hline & $K \rho$ & 22.4 & - \\
\hline & $K_{2}^{*}(1430) \pi$ & 21.7 & - \\
\hline & $K^{*} \rho$ & 17.4 & - \\
\hline & $K \omega$ & 7.07 & - \\
\hline & $K^{*} \omega$ & 6.0 & - \\
\hline & $K^{*} \eta$ & 1.26 & - \\
\hline & $K \phi$ & 0.018 & - \\
\hline & Total & 245 & $\sim 250[1]$ \\
\hline \multirow[t]{22}{*}{$3^{3} S_{1}$} & $K^{*}(1410) \pi$ & 62.3 & - \\
\hline & $K^{*} \pi$ & 44.2 & - \\
\hline & $K \rho$ & 36.8 & - \\
\hline & $K_{2}^{*} \pi$ & 29.4 & - \\
\hline & $K \pi$ & 23.3 & - \\
\hline & $K(1460) \pi$ & 20.8 & - \\
\hline & $K \eta(1295)$ & 18.5 & - \\
\hline & $K a_{2}$ & 15.8 & - \\
\hline & $K \pi(1300)$ & 14 & - \\
\hline & $K \eta$ & 13.1 & - \\
\hline & $K \omega$ & 12 & - \\
\hline & $K_{1}(1650) \pi$ & 11.8 & - \\
\hline & $K b_{1}$ & 11.6 & - \\
\hline & $\eta K_{1}(1400)$ & 7.93 & - \\
\hline & $K_{1} \eta$ & 7.93 & - \\
\hline & $K a_{1}$ & 7.76 & - \\
\hline & $K f_{2}$ & 7.65 & - \\
\hline & $K \omega(1420)$ & 7.62 & - \\
\hline & $K f_{1}(1420)$ & 7.18 & - \\
\hline & $K^{*} \phi$ & 5.54 & - \\
\hline & $K \rho(1450)$ & 5.46 & - \\
\hline & $K h_{1}$ & 4.22 & - \\
\hline
\end{tabular}


Table 5 continued

\begin{tabular}{llll}
\hline States & Channels & This work & Experiment \\
\hline$K_{1}(1400) \pi$ & 3.82 & - \\
$K_{1} \pi$ & 3.82 & - \\
$K^{*} \rho$ & 3.38 & - \\
$K f_{1}$ & 2.92 & - \\
$K \phi$ & 2 & - \\
Total & 393 & - \\
\hline
\end{tabular}

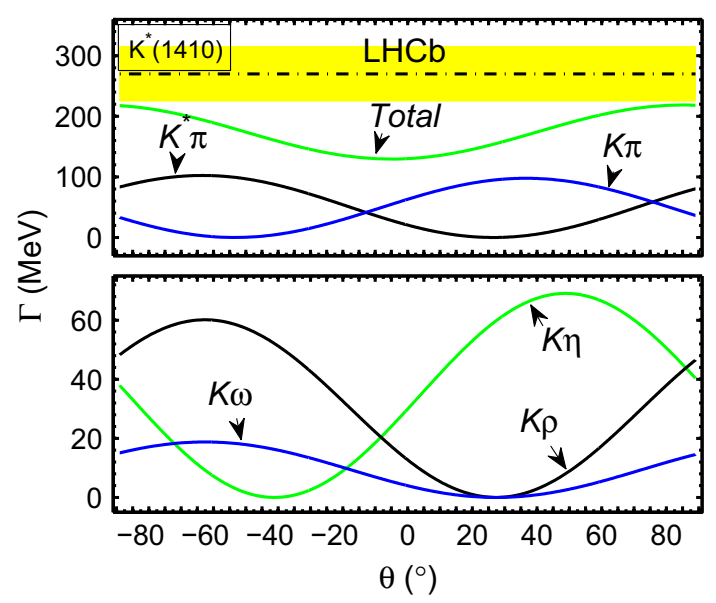

Fig. 1 The $\theta_{s d}$ dependence of the total and partial decay widths of $K^{*}(1410)$. Here, the dot-dashed line is the experimental value from $\mathrm{LHCb}$ [57]

$$
\left(\begin{array}{l}
\left|K^{*}(1410)\right\rangle \\
\left|K^{*}(1680)\right\rangle
\end{array}\right)=\left(\begin{array}{cc}
\cos \theta_{s d} & \sin \theta_{s d} \\
-\sin \theta_{s d} & \cos \theta_{s d}
\end{array}\right)\left(\begin{array}{l}
\left|1^{3} D_{1}\right\rangle \\
\left|2^{3} S_{1}\right\rangle
\end{array}\right)
$$

where $\theta_{s d}$ denotes the mixing angle. In this scenario, we present the decay behavior of $K^{*}(1410)$ dependent on $\theta_{s d}$ as shown in Fig. 1. The result shows that the experimental total width [57] of $K^{*}(1410)$ can be described when $\theta_{s d}$ is taken as $\sim 90^{\circ}$ or $\sim-90^{\circ}$, which supports $K^{*}(1410)$ as a pure $2^{3} S_{1}$ state. We need to emphasize that the branching ratio $\Gamma_{K \pi} / \Gamma_{\text {Total }}$ becomes larger when $\left|\theta_{s d}\right|$ becomes smaller. Thus, the S-D mixing effect on the $K^{*}(1410)$ state is not obvious on describing the experimental data. Of course, we must admit that there still exists a small difference between theoretical and experimental results for the ratio $\Gamma_{K \pi} / \Gamma_{\text {Total }}$.

Finally, we give a conclusion for $K^{*}(1410)$. The mass of $K^{*}(1410)$ as a $2^{3} S_{1}$ state is relatively small, and there exists some disagreement in the branching ratios with experiments. Obviously, confirmation of this state assignment needs more experimental information and theoretical study.

\subsubsection{5 states}

Although $K(1830)$ is not listed in the summary table of the PDG, we still select $K(1830)$ as a possible candidate of the $3^{1} S_{0}$ state and study its decay behavior.

In Table 5, the partial and total decay widths of $K(1830)$ as a $3{ }^{1} S_{0}$ state are shown. Our results show that the largest decay width of $K(1830)$ is given by the channel $K^{*}(1410) \pi$ instead of $K^{*} \rho$ given by Ref. [3]. The other main decay channels contain $K^{*} \pi, K_{0}^{*}(1430) \pi, K \rho, K_{2}^{*}(1430) \pi$, and $K^{*} \rho$. The total width of the theory agrees with the experimental data. Our prediction of the decay information on this state will be helpful for future experimental study, since there exist only two experimental studies on $K(1830)$ until now.

At present, the $3^{3} S_{1}$ state in the kaon family is still absent. Thus, in this work we predict its decay property, where we take the predicted mass of the $3^{3} S_{1}$ state by the MGI model as the input. The results shown in Table 5 indicate that its important decay modes are $\pi K^{*}(1410), K^{*} \pi, K \rho$, and $K_{2}^{*} \pi$, $K \pi, \pi K(1460), K \eta(1295)$. Additionally, $K a_{2}$ also has a sizable contribution to the total width. This predicted decay information is useful to the future experimental search for this missing state.

\section{$4.2 P$-wave kaons}

\subsubsection{P states}

In Table 6, we show the allowed decay channels of $K_{0}^{*}$ (1430), and the corresponding partial and total decay widths. Here, its dominant decay channel of $K_{0}^{*}(1430)$ is $K \pi$, which has decay width $314 \mathrm{MeV}$, which is comparable with the experimental data (267 \pm 36$) \mathrm{MeV}$ listed in PDG [1]. Besides, the $K \eta$ decay channel also has a sizable contribution to the total decay width of $K_{0}^{*}(1430)$. In addition, the obtained total decay width is consistent with the experimental measurement just shown in Table 6 . The above study indicates that the $1^{3} P_{0}$ assignment to $K_{0}^{*}(1430)$ is suitable.

The $K_{2}^{*}(1430)$ together with $a_{2}(1320), f_{2}(1270)$, and $f_{2}^{\prime}(1525)$ may form a $1^{3} P_{2}$ nonet. In Table 6 , we give the partial decay widths of $K_{2}^{*}(1430)$. It dominantly decays into $K \pi$ and $K^{*} \pi$, while the $K \rho, K \omega$, and $K \eta$ modes also have sizable contributions in which $K \eta$ was already observed in experiment [1]. According to Table 6, we can find that our results are consistent with the experimental data. Thus, $K_{2}^{*}(1430)$ as a $1^{3} P_{2}$ state in the kaon family can be supported by our study of its decays.

$K_{1}(1270)$ and $K_{1}(1400)$ as the $1 P$ and $1 P^{\prime}$ states, respectively, satisfy

$$
\left(\begin{array}{l}
\left|K_{1}(1270)\right\rangle \\
\left|K_{1}(1400)\right\rangle
\end{array}\right) \approx\left(\begin{array}{cc}
\cos \theta_{1 P} & \sin \theta_{1 P} \\
-\sin \theta_{1 P} & \cos \theta_{1 P}
\end{array}\right)\left(\begin{array}{l}
\left|1^{1} P_{1}\right\rangle \\
\left|1^{3} P_{1}\right\rangle
\end{array}\right)
$$


Table 6 The decay widths of three $P$-wave states. The unit of the width is $\mathrm{MeV}$

\begin{tabular}{|c|c|c|c|}
\hline States & Channels & This work & Experiment \\
\hline \multirow{3}{*}{$K_{0}^{*}(1430)\left[1^{3} P_{0}\right]$} & $K \pi$ & 314 & $267 \pm 36[58]$ \\
\hline & $\mathrm{K} \eta$ & 2.87 & - \\
\hline & Total & 318 & $270 \pm 80[1]$ \\
\hline \multirow[t]{6}{*}{$K_{2}^{*}(1430)\left[1^{3} P_{2}\right]$} & $K \pi$ & 51.5 & $48.9 \pm 1.7[1,47]$ \\
\hline & $K^{*} \pi$ & 20.4 & $24.8 \pm 1.7[1,47]$ \\
\hline & $K \rho$ & 6.13 & $8.7 \pm 0.8[1,47]$ \\
\hline & $K \omega$ & 1.82 & $2.9 \pm 0.8[1,47,59]$ \\
\hline & $K \eta$ & 0.0665 & $0.15_{-0.1}^{+0.37}[1]$ \\
\hline & Total & 80.1 & $98.5 \pm 2.9[1]$ \\
\hline \multirow[t]{11}{*}{$K_{0}^{*}(1950)\left[2^{3} P_{0}\right]$} & $K \pi$ & 105 & - \\
\hline & $K^{*} \rho$ & 254 & - \\
\hline & $K \pi(1300)$ & 190 & - \\
\hline & $K(1460) \pi$ & 121 & - \\
\hline & $K a_{1}$ & 69.1 & - \\
\hline & $K b_{1}$ & 64.9 & - \\
\hline & $K_{1}(1270) \pi$ & 183 & - \\
\hline & $K_{1}(1270) \eta$ & 6.58 & - \\
\hline & $K_{1}(1400) \pi$ & 5.98 & - \\
\hline & Total & 1000 & $201 \pm 34 \pm 79[58]$ \\
\hline & $\Gamma_{K \pi} / \Gamma_{\text {Total }}$ & $10.5 \%$ & $(52 \pm 8 \pm 12) \%$ \\
\hline
\end{tabular}

where $\theta_{1 P}$ denotes the mixing angle, which leads us to discuss the $\theta_{1 P}$ dependence of the partial and total decay widths of $K_{1}(1270)$ and $K_{1}(1400)$.

According to Fig. 2 which describes the dependence of the mixing angle, $\theta_{1 P}$, of the $K_{1}(1270)$ decay width, we find that $\theta_{1 P}$ should be taken as either $22.5^{\circ}-29^{\circ}$ or $41.5^{\circ}-48^{\circ}$ by fitting the CNTR data of $\Gamma_{K^{*} \pi}$ [55], which is fortunately in the same range when fitted with the ratio $\Gamma\left(K^{*} \pi\right)_{D \text {-wave }} / \Gamma\left(K^{*} \pi\right)_{S \text {-wave }}=1 \pm 0.7$ [55]. Here, the central value of this mixing angle is $\theta_{1 P} \approx 25^{\circ}$ or $45^{\circ}$.

We further investigate the decays of $K_{1}$ (1400). CNTR [55] also gave $\Gamma_{K^{*} \pi} / \Gamma_{\text {Total }}=(94 \pm 6) \%$ [60] for $K_{1}(1400)$, by which we obtain $38^{\circ}<\theta_{1 P}<68^{\circ}$ with central value $\theta_{1 P}=$ $45^{\circ}$, where the details can be found in Fig. 3. ${ }^{2}$ Hence, the above analysis shows that the mixing angle $\theta_{1 P}$ favors $45^{\circ}$, which agrees with the conclusion made in Refs. $[3,47]$ but disagrees with Refs. [61-63], in which they obtained $\theta_{1 P}=$ $34^{\circ}$ and $\sim 60^{\circ}$, respectively.

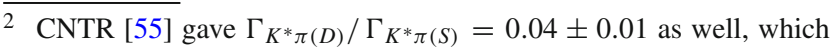
gives the angle range $-7^{\circ}$ to $0^{\circ}$ or $70^{\circ}$ to $78^{\circ}$ if we fit with the ratio, which conflicts with the previous discussion.

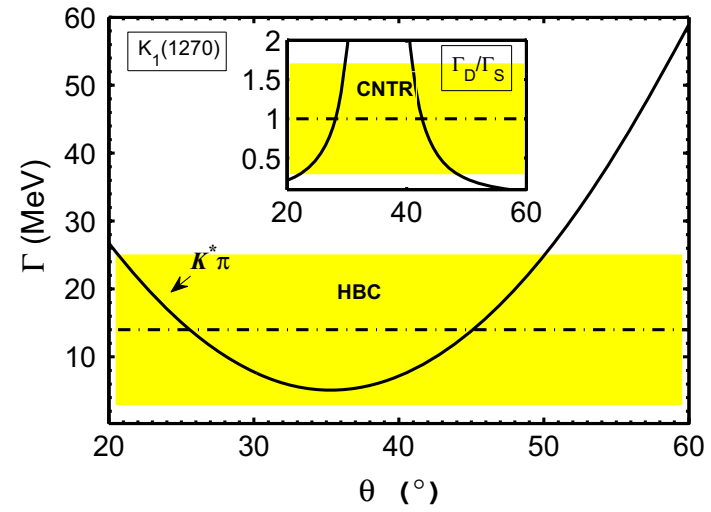

Fig. $2 \theta_{1 P}$ dependence of the $K_{1}(1270) \rightarrow K^{*} \pi$ decay width
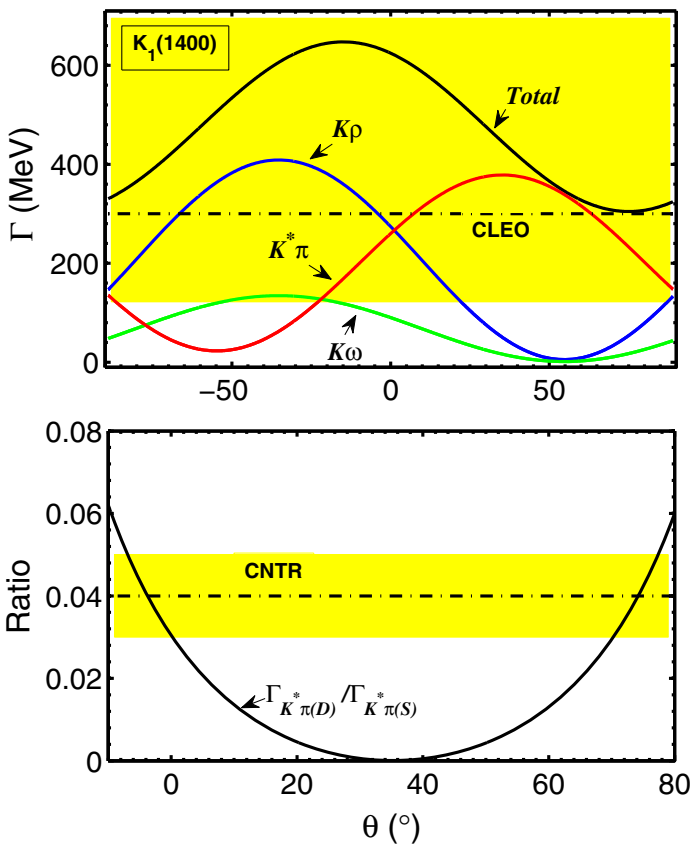

Fig. $3 \theta_{1 P}$ dependence of the partial, the total decay widths and ratio $\Gamma_{K^{*} \pi(D)} / \Gamma_{K^{*} \pi(S)}$ of $K_{1}(1400)$

\subsection{2 $2 P$ states}

As shown in Table 3, Refs. [2-4] and this work give the mass of the $2^{3} P_{0}$ state $1.890,1.791,1.850$, and $1.829 \mathrm{GeV}$, respectively, which are all smaller than the experimental value 1945 $\mathrm{MeV}$ if $K_{0}^{*}(1950)$ is assumed to be a $2^{3} P_{0}$ state. Under the assignment of the $2^{3} P_{0}$ state to $K_{0}^{*}(1950)$, we study the strong decay behavior of $K_{0}^{*}(1950)$, which is presented in Table 6.

Our results show that the $K^{*} \rho$ mode is its dominant decay channel. Its total decay width can reach up to $1000 \mathrm{MeV}$ which is 5 times larger than the experimental value $200 \mathrm{MeV}$. We also notice the result of the total decay width of a $2^{3} P_{0}$ state given by Ref. [3], which is two times larger than the experimental value, where they use a smaller phase space 
Table 7 The strong decay widths of $K_{2}^{*}(1980)$, where the values in brackets and without brackets in the third and fourth columns represent those for $K_{2}^{*}(1980)$ as the $1^{3} F_{2}$ and $2^{3} P_{2}$ states, respectively. The unit of the width is $\mathrm{MeV}$

\begin{tabular}{|c|c|c|c|c|}
\hline States & Channel & This work & Ref. [3] & Experiment \\
\hline \multirow[t]{27}{*}{$K_{2}^{*}(1980)$} & $K_{1}(1270) \pi$ & $36.5(55.7)$ & $6(79)$ & - \\
\hline & $K_{2}(1770) \pi$ & $0.473(5.67)$ & $-(61)$ & \\
\hline & $K b_{1}$ & $24.1(36.7)$ & $8(50)$ & - \\
\hline & $K a_{1}$ & $11.8(10.8)$ & $3(26)$ & - \\
\hline & $K_{1}(1270) \eta$ & $6.31(5.57)$ & $1(22)$ & - \\
\hline & $K \pi$ & $0.788(17.5)$ & $44(20)$ & - \\
\hline & $K h_{1}$ & $11.8(9.00)$ & $4(18)$ & - \\
\hline & $K \rho$ & $10.6(18.3)$ & 44 (13) & - \\
\hline & $K^{*} \pi$ & $10.2(16.2)$ & 47 (13) & - \\
\hline & $K \eta^{\prime}$ & $3.50(6.51)$ & $15(15)$ & - \\
\hline & $K^{*} \eta$ & $24.6(6.80)$ & $26(11)$ & - \\
\hline & $K_{2}^{*}(1425) \pi$ & $61.3(17.4)$ & $15(8)$ & - \\
\hline & $K^{*} \rho$ & $42.1(17.1)$ & $78(8)$ & - \\
\hline & $K f_{1}(1282)$ & $2.93(2.67)$ & $1(7)$ & - \\
\hline & $K a_{2}$ & $28.4(11.8)$ & $3(7)$ & - \\
\hline & $K f_{1}(1426)$ & $0.454(0.32)$ & $-(6)$ & - \\
\hline & $K \phi$ & $11.1(3.31)$ & $12(6)$ & - \\
\hline & $K \omega$ & $3.60(6.09)$ & $14(4)$ & - \\
\hline & $K f_{2}(1270)$ & $12.1(5.55)$ & $3(3)$ & - \\
\hline & $K^{*} \omega$ & $22.0(5.42)$ & 27 (3) & - \\
\hline & $K^{*} \phi$ & $41.0(0.109)$ & $-(1)$ & - \\
\hline & $K_{1}(1400) \pi$ & $7.56(7.32)$ & $11(0)$ & - \\
\hline & $K^{*}(1410) \pi$ & $63.9(2.03)$ & $5(0)$ & - \\
\hline & $\pi K(1460)$ & $27.9(4.31)$ & $2(0)$ & - \\
\hline & $K \pi(1300)$ & $15.0(5.57)$ & $0(0)$ & - \\
\hline & Total & $480(278)$ & $370(283)$ & $373 \pm 33 \pm 60[64](180 \pm 70[54])$ \\
\hline & $\Gamma_{K \rho} / \Gamma_{K * \pi}$ & $1.04(1.14)$ & $0.94(1)$ & $1.49 \pm 0.24 \pm 0.09[58](-)$ \\
\hline
\end{tabular}

(their mass of a $2^{3} P_{0}$ state is $1850 \mathrm{MeV}$ ). We also obtain the branching ratio $\Gamma_{K \pi} / \Gamma_{\text {Total }}=6.4 \%$, which is close to $10.5 \%$ calculated by Ref. [3], but this is smaller than the experimental value $52 \%$. Besides, we also confirm that $K_{1}(1270) \pi$ has sizable contribution to the width of $K_{0}^{*}(1950)$ [3]. It is obvious that there exists a difference between the present theoretical and experimental results. Until now, $K_{0}^{*}(1950)$ has not been established in experiment since this state was omitted from the summary table of PDG [1]. For clarifying it, we suggest further experimental study of $K_{0}^{*}(1950)$, where its resonance parameter and partial decay widths are crucial information.

Then we discuss the possibility of two different assignments to $K_{2}^{*}(1980)$ from two aspects, mass and decay information. In 1987, LASS reported a structure in the reaction $K^{-} p \rightarrow \bar{K}^{0} \pi^{+} \pi^{-} n$ [64], and the obtained resonance parameter are $M=(1973 \pm 8 \pm 25) \mathrm{MeV}$ and $\Gamma=(373 \pm 33 \pm 60) \mathrm{MeV}$. This is the particle called $K_{2}^{*}$ (1980) listed by the PDG [1]. Barnes et al. [3] take the viewpoint that $K_{2}^{*}(1980)$ is a $1^{3} F_{2}$ state, and they give a total width of $300 \mathrm{MeV}$. However, our results show that the mass of a $1^{3} F_{2}$ state is about $2093 \mathrm{MeV}$. Thus, the mass of $K_{2}^{*}(1980)$ is a bit small if $K_{2}^{*}(1980)$ is a $1^{3} F_{2}$ state, which can be supported by another fact, i.e., as an iso-vector $1^{3} F_{2}$ state, $a_{2}$ (2030) is well established in Ref. [32]. In the same $1^{3} F_{2}$ nonet, the meson which contains one $s$ quark is heavier than the mesons which only contain $u / d$ quarks. Along this line, the mass of the $1^{3} F_{2}$ state in the kaon family should be heavier than $2030 \mathrm{MeV}$.

Assuming the $1^{3} F_{2}$ state assignment to $K_{2}^{*}(1980)$, we illustrate its decay behavior. The present work (see Table 7) shows that $K_{1}(1270) \pi$ is the dominant decay channel when we treat $K_{2}^{*}(1980)$ as a $1^{3} F_{2}$ state, even though the channel is not observed in experiments. The $K_{2}(1770) \pi, K b_{1}, K a_{1}$, $K \pi, K \rho$, and $K^{*} \pi$ modes, among which $K \rho$ and $K^{*} \pi$ have been reported in the experiment [1], also have sizable contributions, where we take $\theta_{1 D}=-39^{\circ}$. Our prediction for the channels $K_{1}(1270) \pi, K_{2}(1770) \pi, K b_{1}, K a_{1}$, and $K \pi$ will be helpful for the experimental test of this assignment. 


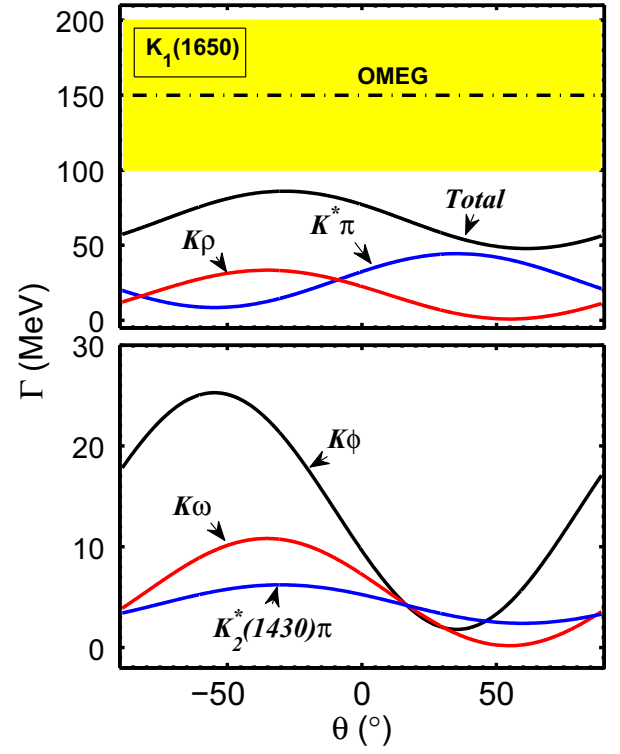

Fig. $4 \theta_{2 P}$ dependence of the decay widths of $K_{1}(1650)$ as a $2 P$ state, where the dot-dashed line is the experimental value of OMEG [65]

Besides the assignment of the $1^{3} F_{2}$ state to $K_{2}^{*}(1980)$, there exists another possibility, $K_{2}^{*}(1980)$ as a $2^{3} P_{2}$ state. The analysis of the mass spectra in Refs. [2-4] and this work shows that the mass of a $2^{3} P_{2}$ state is $1938,1896,1850$, and $1870 \mathrm{MeV}$, respectively. Thus, the experimental mass value of $K_{2}^{*}(1980)$ is a bit larger than a $2^{3} P_{2}$ state. If $K_{2}^{*}(1980)$ is a $2^{3} P_{2}$ state, its main decay modes are $K^{*} \rho, K \pi, K^{*} \pi, K \rho$, $K^{*} \eta, K \eta^{\prime}$, and $K^{*} \omega$. Besides the $K \rho$ and $K^{*} \pi$ modes, one notices that $K f_{2}$ has been observed in experiments, which has a sizable contribution in theory. Hence, $K_{2}^{*}(1980)$ as a $2^{3} P_{2}$ state is also a possible assignment.

Just presented above, we discuss two assignments to $K_{2}^{*}(1980)$, where the decay behaviors of $K_{2}^{*}(1980)$ under two assignments are different. Thus, we should combine further experimental decay information of $K_{2}^{*}(1980)$ with our results to determine which possibility of its assignments we should take.

The $K_{1}(1650)$ and its partner $K_{1}\left(2 P^{\prime}\right)$ satisfy

$$
\left(\begin{array}{l}
\left|K_{1}(1650)\right\rangle \\
\left|K_{1}(2030)\right\rangle
\end{array}\right) \approx\left(\begin{array}{cc}
\cos \theta_{2 P} & \sin \theta_{2 P} \\
-\sin \theta_{2 P} & \cos \theta_{2 P}
\end{array}\right)\left(\begin{array}{l}
\left|2^{1} P_{1}\right\rangle \\
\left|2^{3} P_{1}\right\rangle
\end{array}\right)
$$

In Fig. 4, we show the partial and total decay widths of $K_{1}(1650)$ depending on the mixing angle $\theta_{2 P}$ if the mass of $K_{1}(1650)$ is adored to be $M=1650 \pm 50 \mathrm{MeV}$ [1]. Since the decays of $K_{1}(1650)$ into $K \pi \pi$ and $K \phi$ were observed in experiments [55,65-67], we can roughly conclude that $\theta_{2} P$ is probably less than zero as seen from Fig. 4 , where $K \rho, K \phi$ and $K^{*} \pi$ have sizable contributions to the total decay width of $K_{1}(1650)$ in our calculation.

In experiment, $K_{1}(1650)$ is also not well established since this state is omitted from the summary table of the PDG [1].

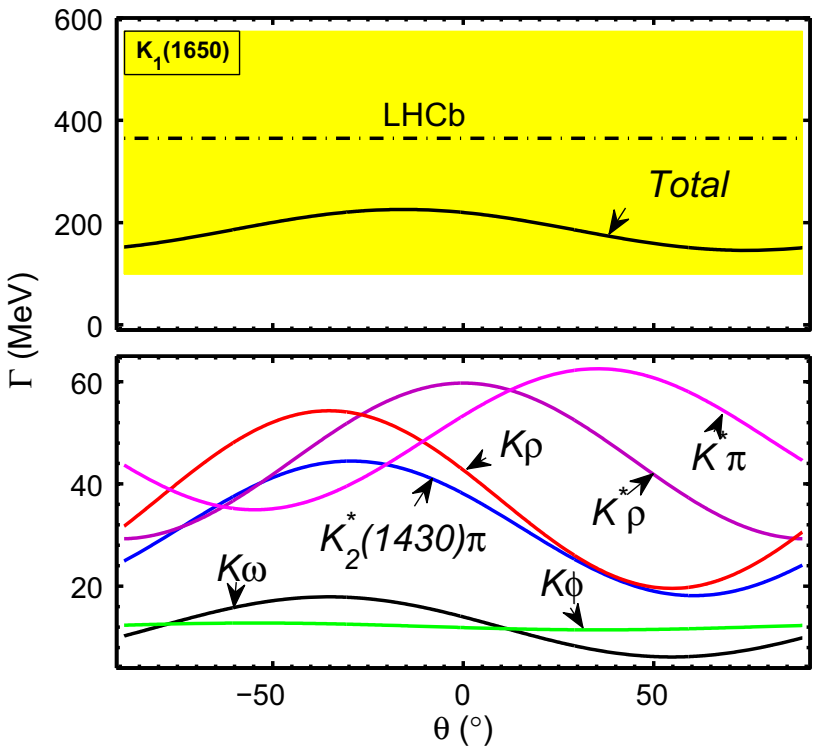

Fig. $5 \theta_{2 P}$ dependence of the decay widths of $K_{1}(1650)$ as a $2 P$ state with the measured center mass of $1793 \mathrm{MeV}$ from LHCb [66], where the dot-dashed line is the experimental value of $\mathrm{LHCb}$ [66]

More experimental and theoretical efforts are necessary to establish $K_{1}(1650)$. We notice new experimental information of $K_{1}$ (1650) from LHCb [66], where the measured mass of $K_{1}(1650)$ is $\left(1793 \pm 59_{-101}^{+153}\right) \mathrm{MeV}$, which is about $150 \mathrm{MeV}$ larger than the experimental data given by Ref. [1]. Taking the $\mathrm{LHCb}$ mass result as an input, we investigate the strong decay behaviors of $K_{1}(1650)$ again, which are shown in Fig. 5. Here, $K^{*} \pi, K^{*} \rho, K \rho$ and $K_{2}^{*}(1430) \pi$ are dominant decay channels. However, we cannot give a further constraint on the mixing angle $\theta_{2 P}$ by comparing an experimental width with our theoretical result due to a large experimental error of the $\mathrm{LHCb}$ experimental data.

In the following, we discuss the partner of $K_{1}$ (1650). Reference [68] gives the following equation for the mass relation between the pure states and physical state:

$m_{K_{1}\left(2^{3} P_{1}\right)}^{2}=\cos \left(\theta_{2 P}\right)^{2} m_{K_{1}\left(2 P^{\prime}\right)}^{2}+\sin \left(\theta_{2 P}\right)^{2} m_{K_{1}(2 P)}^{2}$,

$m_{K_{1}\left(2^{1} P_{1}\right)}^{2}=\sin \left(\theta_{2 P}\right)^{2} m_{K_{1}\left(2 P^{\prime}\right)}^{2}+\cos \left(\theta_{2 P}\right)^{2} m_{K_{1}(2 P)}^{2}$.

Substituting $m_{K_{1}\left(2^{1} P_{1}\right)}=1840 \mathrm{MeV}$ and $m_{K_{1}\left(2^{3} P_{1}\right)}=1861$ $\mathrm{MeV}$ given in Table 3 into Eq. (42), we obtain the mass of the $K_{1}(1650)$ partner, $m_{K_{1}\left(2 P^{\prime}\right)}$, about $2030 \mathrm{MeV}$ and the $\theta_{2 P} \approx$ $\pm 43^{\circ}$ if taking the mass of $K_{1}(1650)$ as $M=(1650 \pm 50)$ $\mathrm{MeV}$ [1]. Based on the above analysis of $K_{1}(1650)$, we suggest $\theta_{2 P} \approx-43^{\circ}$. If taking the LHCb mass measurement [66] of $K_{1}(1650)$ as an input, the mass of the $K_{1}(1650)$ partner is estimated to be $1906 \mathrm{MeV}$. In Ref. [66], the resonance parameters of the $K_{1}(1650)$ partner are given, i.e., $M=\left(1968 \pm 65_{-172}^{+70}\right) \mathrm{MeV}$ and $\Gamma=\left(396 \pm 170_{-178}^{+174}\right)$ $\mathrm{MeV}$. Considering the present status, we select the experimental mass (1968 MeV [66]) for the partner of $K_{1}(1650)$ 
Table 8 The main strong decay widths of the $K_{1}\left(2 P^{\prime}\right)$ state which as the partner of $K_{1}(1650)$. Here, the mass of $K_{1}\left(2 P^{\prime}\right)$ is taken as 1968 $\mathrm{MeV}$ [66]. $c=\cos \theta_{2 P}$ and $s=\sin \theta_{2 P}$. The unit of the width is $\mathrm{MeV}$

\begin{tabular}{ll}
\hline Decay channel & Width \\
\hline Total & $440 c^{2}+70.2 \mathrm{c} \mathrm{s}+385 s^{2}$ \\
$K^{*} \rho$ & $166 c^{2}+111 s^{2}$ \\
$K_{2}^{*} \pi$ & $71.5 c^{2}+57 \mathrm{c} \mathrm{s}+111 s^{2}$ \\
$\mathrm{~K} a_{2}$ & $59 c^{2}+48 . \mathrm{c} \mathrm{s}+80.8 \mathrm{~s}$ \\
$K^{*} \pi$ & $75.4 c^{2}+8.84 \mathrm{c} \mathrm{s}+72.3 s^{2}$ \\
$\mathrm{~K} \rho$ & $63.1 c^{2}+2.81 \mathrm{c} \mathrm{s}+64.1 s^{2}$ \\
$K^{*} \omega$ & $53.8 c^{2}+36 s^{2}$ \\
$\mathrm{~K} f_{2}$ & $22.4 c^{2}+10.5 \mathrm{c} \mathrm{s}+20.8 s^{2}$ \\
\hline
\end{tabular}

when discussing the decay behavior of the $K_{1}\left(2 P^{\prime}\right)$ state just shown in Table 8. Here, the calculated width of $K_{1}\left(2 P^{\prime}\right)$ is about (440-570) $\mathrm{MeV}$, which is comparable to the experimental data [66]. Its main decay modes are $K^{*} \rho, K_{2}^{*} \pi, K a_{2}$, $K^{*} \pi, K \rho, K^{*} \omega$, and $K f_{2}$.

\section{$4.3 D$-wave kaons}

\subsubsection{D states}

$K^{*}(1680)$ together with $\rho(1700)$ and $\omega(1650)$ forms a $1^{3} D_{1}$ nonet. Barnes et al. [3] predicted that this state should have a mass of $1850 \mathrm{MeV}$, but we obtain $1.766 \mathrm{GeV}$, which is closer to the experimental value $1.717 \mathrm{GeV}$. The mass spectrum analysis supports $K^{*}(1680)$ as a $1^{3} D_{1}$ state.

As shown in Table $9, K^{*}(1680)$ as a pure $1^{3} D_{1}$ state mainly decays into final states $K_{1}(1270) \pi, K h_{1}, K \pi$, and $K \eta$, while the $K^{*} \pi$ and $K \rho$ modes also have sizable contributions. We notice that the obtained ratios of partial decay widths of $K \pi, K^{*} \pi$, and $K \rho$ in this work are comparable with experimental data given in PDG. Since the branching ratios of the $K \pi, K^{*} \pi$, and $K \rho$ decay channels given by PDG are $38.7,29.9$, and $31.4 \%$, we conclude that the remaining $K_{1}(1270) \pi$ decay channel has a very small width. However, our calculation shows that $K_{1}(1270) \pi$ is a main contribution to the total width which is consistent with the conclusion from the former analysis in [3] but contradicts the present experimental data. Here we and the authors of Ref. [3] adopted the mixing angle $\theta_{1 P}=45^{\circ}[4,47]$ in the corresponding calculations. It is obvious that we need to face this puzzle in this channel. More experimental and theoretical efforts are needed to clarify this point.

$K_{2}(1770)$ and $K_{2}(1820)$ satisfy

$$
\left(\begin{array}{l}
\left|K_{2}(1770)\right\rangle \\
\left|K_{2}(1820)\right\rangle
\end{array}\right) \approx\left(\begin{array}{cc}
\cos \theta_{1 D} & \sin \theta_{1 D} \\
-\sin \theta_{1 D} & \cos \theta_{1 D}
\end{array}\right)\left(\begin{array}{l}
\left|1^{1} D_{2}\right\rangle \\
\left|1^{3} D_{2}\right\rangle
\end{array}\right)
$$

According to Fig. 6, we find that $K_{2}(1770)$ mainly decays to $K_{2}^{*}(1430) \pi, K^{*} \pi, K \pi$, and $K \omega$. Experiments show that $K_{2}^{*}(1430) \pi$ is the dominant decay mode of $K_{2}(1770)$ [1], which indicates that $\theta_{1 D}$ favors the value less than zero.

$K_{3}^{*}(1780)$ together with $\rho_{3}(1690), \omega_{3}(1670)$, and $\phi_{3}(1850)$ forms a $1^{3} D_{3}$ nonet. We give it a mass of $1.781 \mathrm{GeV}$ by the MGI model, which is consistent with the experimental data of $1.776 \mathrm{GeV}$. As shown in Table 9, even though $K^{*} \rho$ is the dominant decay mode of $K_{3}^{*}(1780)$, it is not observed in experiments so far. The channel $K^{*} \omega$ has a sizable contribution to its total decay width, which is still missing in experiment. A final state $K \pi$ largely contributes to the total width and theory and experiments are consistent. The branching ratio $\Gamma_{K^{*} \pi} / \Gamma_{K \pi}$ agrees with the experimental data [70].

Next, let us focus on $K_{2}(1820)$. According to Fig. 7, one notices that $K_{2}(1820)$ probably decays to $K \pi \pi, K_{2}^{*}(1430) \pi$, $K f_{2}(1270), K^{*} \pi$, and $K \omega$, in which $K \pi \pi$ comes from $K \rho$ channel. As seen from the $\theta_{1 D}$ dependence of the widths of $K_{2}(1820)$ in Fig. 7, we notice that contributions of $K \rho$ and $K f_{2}(1270)$ are large when $\theta_{1 D}<0$, which indicates that it is very likely that $\theta_{1 D}$ is smaller than zero, which is consistent with the previous analysis for $K_{2}(1770)$. Because of the absence of the experimental information, we cannot confirm the angle $\theta_{1 D}$, while our results will be helpful for the future experiments to study this state.

\subsubsection{D states}

As one of the $2 D$ states is missing, using the familiar program with $2 P$ states, we obtain

$$
\left(\begin{array}{l}
\left|K_{2}(1990)\right\rangle \\
\left|K_{2}(2250)\right\rangle
\end{array}\right) \approx\left(\begin{array}{cc}
\cos \theta_{2 D} & \sin \theta_{2 D} \\
-\sin \theta_{2 D} & \cos \theta_{2 D}
\end{array}\right)\left(\begin{array}{l}
\left|2^{1} D_{2}\right\rangle \\
\left|2^{3} D_{2}\right\rangle
\end{array}\right),
$$

where $K_{2}(1990)$ is obtained from an equation similar to Eq. (42).

According to Table 10, one finds that $K_{2}(2250)$ as a $2 D^{\prime}$ state mainly decays into $K a_{2}(1700), K_{2}^{*}(1980) \pi$, $K_{3}^{*}(1780) \pi, K^{*}(1410) \pi$, and $K_{2}^{*} \pi . K_{2}^{*} \pi$ and $K f_{2}(1270)$ have been observed in experiments which have sizable contributions to the total width. Besides, $K \rho$, which is an important decay channel in our result, can decay into $K \pi \pi$, which is observed in experiment. On the other hand, the theoretical total width is larger than the experimental value $180 \mathrm{MeV}$ given in PDG. We need more experimental information to study this $2 D^{\prime}$ state, to test our results, and to have more detailed decay widths to ascertain the value of $\theta_{2 D}$.

We use $1994 \mathrm{MeV}$ as the mass of the partner of $K_{2}(2250)$ with $\theta_{2 D} \approx \pm 44^{\circ}$, and calculate the strong decay of this state as shown in Table 11. According to this table, we can find that its main decay channels are $K^{*} \pi, K \rho, K_{2}^{*} \pi$, and $K_{3}^{*}(1780) \pi$. 
Table 9 The decay widths of the $1^{3} D_{1}$ and $1^{3} D_{3}$ states. Here, $K^{*}(1680)$ and $K_{3}^{*}(1780)$ are assigned to be the pure $1^{3} D_{1}$ state and $1^{3} D_{3}$ states, respectively. The unit of the width is $\mathrm{MeV}$

\begin{tabular}{|c|c|c|c|c|}
\hline State & Channel & This work & Ref. [3] & Experiment \\
\hline \multirow[t]{14}{*}{$1^{3} D_{1}$} & $K \pi$ & 69.2 & 45 & - \\
\hline & $K^{*} \pi$ & 41.8 & 25 & - \\
\hline & $K \rho$ & 44.7 & 26 & - \\
\hline & $K \eta$ & 64.4 & 53 & - \\
\hline & $K^{*} \rho$ & 6.33 & 2 & - \\
\hline & $K^{*} \omega$ & 1.69 & 1 & - \\
\hline & $K h_{1}$ & 78 & 33 & - \\
\hline & $K_{1}(1270) \pi$ & 330 & 145 & - \\
\hline & $K_{1}(1400) \pi$ & 7.86 & 0 & - \\
\hline & $K \phi$ & 9.35 & 45 & - \\
\hline & Total & 653 & 348 & $426 \pm 18 \pm 30[58]$ \\
\hline & $\Gamma_{K \pi} / \Gamma_{K^{*} \pi}$ & 1.66 & 1.8 & $2.8 \pm 1.1[71]$ \\
\hline & $\Gamma_{K \rho} / \Gamma_{K \pi}$ & 0.65 & 0.58 & $1.2 \pm 0.4[71]$ \\
\hline & $\Gamma_{K \rho} / \Gamma_{K^{*} \pi}$ & 1.07 & 1.04 & $1.05_{-0.11}^{+0.27}$ \\
\hline \multirow[t]{18}{*}{$1^{3} D_{3}$} & $K^{*} \rho$ & 118 & 42 & - \\
\hline & $K \rho$ & 20.1 & 10 & $74 \pm 10[47]$ \\
\hline & $K^{*} \omega$ & 36.4 & 12 & - \\
\hline & $K \pi$ & 38.1 & 40 & $31.7 \pm 3.7[47]$ \\
\hline & $K^{*} \pi$ & 28.5 & 14 & $45 \pm 7[47]$ \\
\hline & $K \omega$ & 6.45 & 3 & - \\
\hline & $K \eta$ & 9.67 & 19 & $48 \pm 21[1], 15 \pm 6[72]$ \\
\hline & $K_{1}(1270) \pi$ & 1.68 & 1 & - \\
\hline & $K_{1}(1400) \pi$ & 2.80 & 1 & - \\
\hline & $K_{2}^{*}(1430) \pi$ & 4.18 & 1 & $<25[72]$ \\
\hline & Total & 266 & 145 & $225 \pm 60[73]$ \\
\hline & $\Gamma_{K \rho} / \Gamma_{K * \pi}$ & 0.702 & 0.71 & $1.52 \pm 0.23[70]$ \\
\hline & $\Gamma_{K^{*} \pi} / \Gamma_{K \pi}$ & 0.748 & 0.35 & $1.09 \pm 0.26[70]$ \\
\hline & $\Gamma_{K \eta} / \Gamma_{K \pi}$ & 0.253 & 0.48 & $1.6 \pm 0.7[1]$ \\
\hline & $\Gamma_{K \pi} / \Gamma_{\text {Total }}$ & 0.143 & 0.28 & $0.188 \pm 0.010[1]$ \\
\hline & $\Gamma_{K \rho} / \Gamma_{\text {Total }}$ & $7.5 \%$ & $6.9 \%$ & $(31 \pm 9) \%[1]$ \\
\hline & $\Gamma_{K^{*} \pi} / \Gamma_{\text {Total }}$ & $10.7 \%$ & $9.7 \%$ & $(20 \pm 5) \%[1]$ \\
\hline & $\Gamma_{K \eta} / \Gamma_{\text {Total }}$ & $3.6 \%$ & $13 \%$ & $(30 \pm 13) \%[1]$ \\
\hline
\end{tabular}

\section{$4.4 F$-wave kaons}

\subsection{1 $1 F$ states}

In this section, we discuss possibility of different assignments of $K_{2}^{*}$ (1980) from two aspects, mass and decay information. In 1987, LASS reported a structure in the reaction $K^{-} p \rightarrow$ $\bar{K}^{0} \pi^{+} \pi^{-} n$ [64], and they obtained the resonance parameters $M=(1973 \pm 8 \pm 25) \mathrm{MeV}$ and $\Gamma=(373 \pm 33 \pm 60)$ $\mathrm{MeV}$. This is the particle called $K_{2}^{*}(1980)$ listed in PDG [1]. Barnes et al. [3] have the viewpoint that $K_{2}^{*}(1980)$ is a $1^{3} F_{2}$ state, and they give a total width of $300 \mathrm{MeV}$. On the other hand, our results show that the mass of a $1^{3} F_{2}$ state is about $2093 \mathrm{MeV}$. Ebert et al. [4] predict a $1^{3} F_{2}$ state with a mass of $1964 \mathrm{MeV}$. As the partner of an iso-vector of $1^{3} F_{2}$, $a_{2}$ (2030) is well established in Ref. [32]. In the same nonet, the meson which contains one $s$ quark is probably heavier than the mesons which only contain $u / d$ quarks. Along this line, the mass of the $1^{3} F_{2}$ state of the kaon should be larger than $2030 \mathrm{MeV}$, so that the mass $K_{2}^{*}(1980)$ is a bit small as a $1^{3} F_{2}$ state. References [2-4] and this work give the mass for a $2^{3} P_{2}$ state $1938,1896,1850$, and $1870 \mathrm{MeV}$, respectively, and for its iso-vector partner, $a_{2}$ (1700) [32], the mass of $K_{2}^{*}(1980)$ is a bit larger as a $2^{3} P_{2}$ state. We should, of course, combine the decay information of $K_{2}^{*}(1980)$ to determine which possibility for its assignment we should take.

Both Ref. [3] and this work (see Table 7) show that $K_{1}(1270) \pi$ is the dominant decay channel when we treat $K_{2}^{*}(1980)$ as a $1^{3} F_{2}$ state, even though the channel is not observed in experiments. $K_{2}(1770) \pi, K b_{1}, K a_{1}, K \pi, K \rho$, 


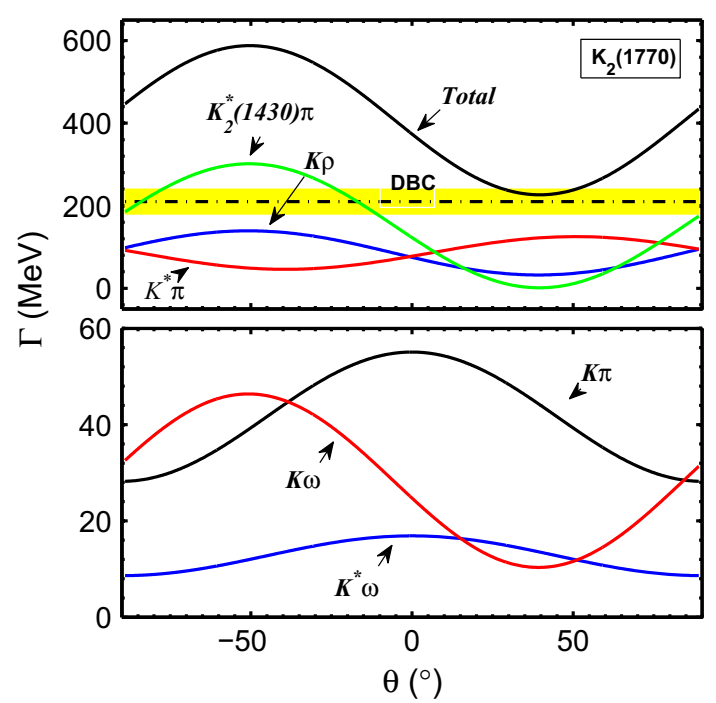

Fig. $6 \theta_{1 D}$ dependence of the width of $K_{2}(1770)$, where the dotdashed line is the experimental value of DBC [69]
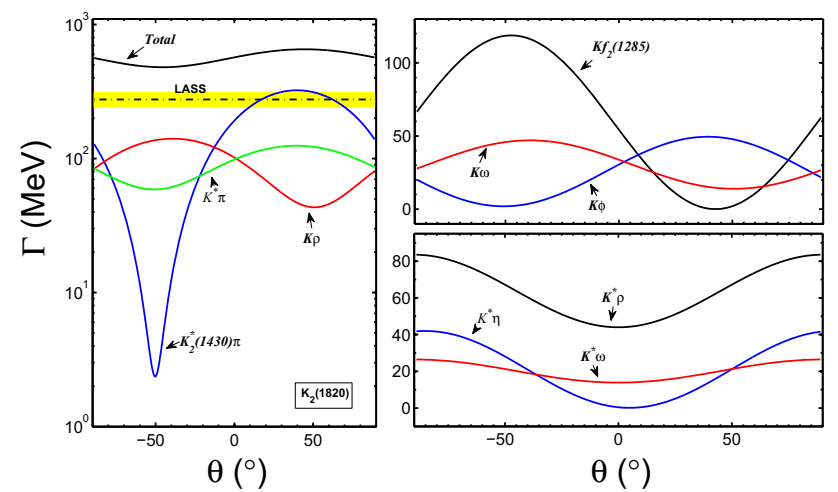

Fig. $7 \theta_{1 D}$ dependence of the decay width of $K_{2}(1820)$. Here a dotdashed line LASS with a yellow band is taken from [64]

and $K^{*} \pi$ modes, among which $K \rho$ and $K^{*} \pi$ have been reported in the experiment [1], also have sizable contributions, where we take $\theta_{1 D}=-39^{\circ}$. If $K_{2}^{*}(1980)$ is a $1^{3} F_{2}$ state, our prediction for the channels $K_{1}(1270) \pi$, $K_{2}(1770) \pi, K b_{1}, K a_{1}$, and $K \pi$ will be helpful for the experiment to test this assignment.

Both Ref. [3] and our results show that $K_{2}^{*}(1980)$ is $2^{3} P_{2}$ state, where Ref. [3] takes the mass to be $1850 \mathrm{MeV}$ and we take an experimental value of $1973 \mathrm{MeV}$. For this reason, the results between their and this work show some difference. The main decay modes are $K^{*} \rho, K \pi, K^{*} \pi, K \rho, K^{*} \eta, K \eta^{\prime}$, and $K^{*} \omega$. Besides $K \rho$ and $K^{*} \pi$, one notices that $K f_{2}$ has been observed in experiments which has sizable contributions in theory. Hence $K_{2}^{*}(1980)$ to be assigned to $2^{3} P_{2}$ is also reasonable.

Finally, let us draw a rough conclusion for $K_{2}^{*}(1980)$. According to the mass analysis, the mass of $K_{2}^{*}(1980)$ is a bit small when assigned to a $1^{3} F_{2}$ state and a bit large when assigned to a $2^{3} P_{2}$ state. According to the decay information, $K_{2}^{*}(1980)$ is in favor of a $2^{3} P_{2}$ state. We still, however, need more experimental information to test our assignment for $K_{2}^{*}(1980)$. What is more important is that we give the prediction that the partial widths of $K_{2}^{*}(1425) \pi, K^{*}(1410) \pi$, $K^{*} \omega, K^{*} \rho$, and $K^{*} \eta$ treating $K_{2}^{*}(1980)$ as $1^{3} F_{2}$ will be much larger than those of the case of a $2^{3} P_{2}$ state. The experimental study of these decay modes combined with our prediction will help us to determine the assignment of $K_{2}^{*}(1980)$. Besides the above, our prediction can help future experiments to find the missing $1^{3} F_{2}$ or $2^{3} P_{2}$ state. According to Table 3, one can notice that our spectral results are consistent with the mass of $K_{4}^{*}(2045)$ given by PDG when we treat it as $1^{3} F_{4}$ state. As for the strong decay of $K_{4}^{*}(2045)$, one can notice that both the results of Ref. [3] and this work (Table 12) show that $K^{*} \rho, K \pi, K^{*} \pi, K^{*} \omega$, and $K \rho$ are the main decay channels. The PDG gives two partial width ratios: one is $\Gamma_{K \pi} / \Gamma_{\text {Total }}=(9.9 \pm 1.2) \%$ and our result is $\Gamma_{K \pi} / \Gamma_{\text {Total }}=8.4 \%$, which is consistent with the experiment. Another is $\Gamma_{K^{*} \phi} / \Gamma_{\text {Total }}=(1.4 \pm 0.7) \%$ and we obtain $\Gamma_{K^{*} \phi} / \Gamma_{\text {Total }}=1.54 \%$, which is consistent with the experiment as well. On the other hand, Ref. [3] obtained 21 and $3.1 \%$ for these two partial width ratios, which are different from the experiment. These results, of course, prove the superiority of the accurate meson wave functions we have obtained.

References [2-4] and this work give the mass of a $1 F$ state 2131, 2009, 2050, and $2075 \mathrm{MeV}$ (which we call $K_{3}$ (2075), and strictly speaking, this state is a pure $1^{1} F_{3}$ state, here we assume the physical state $1 F$ has this mass), respectively, among which one notices that the last two results are almost identical. $K_{3}(2075)$ is assigned to the missing $1 F$ state. We present the $\theta_{n F}$ dependence of the widths for these two cases in Table 13. The total width of a $1 F$ state with a mass of $2075 \mathrm{MeV}$ is about (400-600) MeV, which means that the predicted $K_{3}(2075)$ is a broad state and it is not easy to identify $K_{3}(2075)$ in experiments. Its main decay channels are $K_{3}^{*}(1780) \pi, K^{*} \rho, K^{*} \pi, K a_{2}, K \rho$ and $K_{2}^{*} \pi$.

\subsection{2 $2 F$ states}

$K_{3}(2 F)$ and $K_{3}\left(2 F^{\prime}\right)$ mixing satisfies

$$
\left(\begin{array}{l}
\left|K_{3}(2320)\right\rangle \\
\left|K_{3}(2360)\right\rangle
\end{array}\right) \approx\left(\begin{array}{cc}
\cos \theta_{2 F} & \sin \theta_{2 F} \\
-\sin \theta_{2 F} & \cos \theta_{2 F}
\end{array}\right)\left(\begin{array}{l}
\left|2^{1} F_{3}\right\rangle \\
\left|2^{3} F_{3}\right\rangle
\end{array}\right)
$$

where $K_{3}(2360)$ is obtained from an equation similar to Eq. (42). The total width of $K_{3}(2320)$ is nearly (180-200) $\mathrm{MeV}$, which is consistent with the data of OMEG (150 \pm 30) $\mathrm{MeV}$ [75]. $K_{3}(2320)$ mainly decays to $K_{3}^{*}(1780) \pi$, $K^{*}(1410) \pi, K \rho$ and $K^{*} \pi$. The total width of $K_{3}(2360)$ is nearly $(80-120) \mathrm{MeV} . \quad K_{3}(2360)$ mainly decays to $K_{3}^{*}(1780) \pi, K \rho_{3}(1690), K \rho$ and $K^{*} \pi$, which are given in 
Table 10 Strong decay information of $K_{2}(2250)\left(2 D^{\prime}\right)$, where $s$ and $c$ represent sine and cosine functions. The unit of the width is $\mathrm{MeV}$

\begin{tabular}{llll}
\hline Decay channel & Width & Decay channel & Width \\
\hline Total & $956 c^{2}-267 c s+1044 s^{2}$ & $K a_{2}(1700)$ & $115 c^{2}-188 c s+77.1 s^{2}$ \\
$K_{2}^{*}(1980) \pi$ & $82.6 c^{2}-136 c s+57.4 s^{2}$ & $K_{3}^{*}(1780) \pi$ & $67.3 c^{2}-135 c s+71.4 s^{2}$ \\
$K^{*}(1410) \pi$ & $75.5 s^{2}+55.6 c^{2}-97.2 c s$ & $K_{2}^{*} \pi$ & $58.9 c^{2}-70.9 c s+64.2 s^{2}$ \\
$K a_{2}$ & $52.9 c^{2}-57 c s+55.1 s^{2}$ & $K \rho_{3}(1690)$ & $33.7 c^{2}-64.5 c s+31 s^{2}$ \\
$K^{*} \pi$ & $31.3 c^{2}-50.3 c s+21.1 s^{2}$ & $K \rho(1450)$ & $42.4 c^{2}-23.3 c s+37.6 s^{2}$ \\
$K \rho$ & $29.7 c^{2}-48 c s+19.9 s^{2}$ & $K^{*} a_{2}$ & $9.24 c^{2}-30.9 c s+37.9 s^{2}$ \\
$K^{*}(1410) \rho$ & $22.1 c^{2}+42.1 s^{2}$ & $K^{*} \pi(1300)$ & $25.6 c^{2}-40.4 c s+17.3 s^{2}$ \\
$K^{*} \rho$ & $37.7 c^{2}+40.2 s^{2}$ & $K_{1}(1400) \rho$ & $15.5 c^{2}-42.8 c s+16.7 s^{2}$ \\
$K \omega$ & $15.1 c^{2}-29 c s+14 s^{2}$ & $K f_{2}$ & $19.9 c^{2}-18.1 c s+20.1 s^{2}$ \\
$K f_{2}^{\prime}(1525)$ & $19.9 c^{2}-18.1 c s+20.1 s^{2}$ & $K_{2}^{*} \omega$ & $3.49 c^{2}-12.9 c s+15.6 s^{2}$ \\
$K \omega$ & $9.85 c^{2}-15.9 c s+6.61 s^{2}$ & $K^{*} b_{1}$ & $14.3 c^{2}-0.4 c s+16.1 s^{2}$ \\
$K^{*} a_{1}$ & $6.74 c^{2}-7 c s+14.1 s^{2}$ & $K a_{0}(1450)$ & $4.36 c^{2}-13.7 c s+10.8 s^{2}$ \\
$K \omega(1420)$ & $14.1 c^{2}-2.21 c s+13.6 s^{2}$ & $K^{*} \eta$ & $0.101 c^{2}-0.517 c s+14.2 s^{2}$ \\
$K^{*} \omega$ & $12.5 c^{2}+13.2 s^{2}$ & $K^{*}(1410) \omega$ & $6.37 c^{2}+12.3 s^{2}$ \\
$K^{*} h_{1}$ & $10.2 c^{2}-3.25 c s+10.6 s^{2}$ & $K^{*} f_{2}$ & $6.31 c^{2}-7.46 c s+9.34 s^{2}$ \\
$K^{*}(1680) \pi$ & $4.98 c^{2}-11 c s+6.44 s^{2}$ & $K^{*} \eta(1295)$ & $0.0195 c^{2}-0.914 c s+10.7 s^{2}$ \\
$K^{*} \phi$ & $8.35 c^{2}+6.81 s^{2}$ & $K_{1}(1400) \omega$ & $1.26 c^{2}-1.09 c s+8.18 s^{2}$ \\
\hline
\end{tabular}

Table 11 The main strong decay widths of $K_{2}(1990)$ as an $2 D$ state, where $s$ and $c$ represent sine and cosine functions. The unit of the width is $\mathrm{MeV}$

\begin{tabular}{ll}
\hline Decay channel & Width \\
\hline Total & $97.9 c^{2}-17.3 c s+120 s^{2}$ \\
$K^{*} \pi$ & $15.1 c^{2}-22 c s+19.6 s^{2}$ \\
$\mathrm{~K} \rho$ & $12.5 c^{2}+19.2 c s+16.4 s^{2}$ \\
$K_{2}{ }^{*} \pi$ & $17.9 c^{2}-16.5 c s+7.59 s^{2}$ \\
$K_{3}^{*}(1780) \pi$ & $7.16 c^{2}-14.4 c s+7.26 s^{2}$ \\
\hline
\end{tabular}

Table 14. Although we cannot give the mixing angle of these two states for lack of experimental information, our theoretical results can be helpful for studying these two states in future experiments.

\section{$4.5 G$-wave kaons}

\subsection{1 $1 G$ states}

$K_{4}(1 G)$ and $K_{4}\left(1 G^{\prime}\right)$ mixing satisfies

$$
\left(\begin{array}{c}
\left|K_{4}(2310)\right\rangle \\
\left|K_{4}\left(1 G^{\prime}\right)\right\rangle
\end{array}\right) \approx\left(\begin{array}{cc}
\cos \theta_{1 G} & \sin \theta_{1 G} \\
-\sin \theta_{1 G} & \cos \theta_{1 G}
\end{array}\right)\left(\begin{array}{l}
\left|1^{1} G_{4}\right\rangle \\
\left|1^{3} G_{4}\right\rangle
\end{array}\right)
$$

We assume the mass of a $1 G$ state to be about $2309 \mathrm{MeV}$, which we call $K_{4}(2310)$. The GI model [2] and Ref. [4] give this mass values of 2422 and $2255 \mathrm{MeV}$, respectively, while the mass of the $K_{4}$ state in PDG is $2490 \mathrm{MeV}$. According to Table $3, K_{4}(2500)$ may be a $2 G$ state. We predict the
Table 12 The strong decay widths of $K_{4}^{*}(2045)$ assigned to a $1^{3} F_{4}$ state. The unit of the width is $\mathrm{MeV}$

\begin{tabular}{|c|c|c|c|c|}
\hline State & Channel & This work & Ref. [3] & Experiment \\
\hline \multirow[t]{19}{*}{$K_{4}^{*}(2045)\left(1^{3} F_{4}\right)$} & $K^{*} \rho$ & 84.9 & 29 & - \\
\hline & $K \rho$ & 16.1 & 7 & - \\
\hline & $K^{*} \omega$ & 27.7 & 9 & - \\
\hline & $K \omega$ & 5.24 & 2 & - \\
\hline & $K \pi$ & 21.0 & 21 & - \\
\hline & $K^{*} \pi$ & 20.5 & 8 & - \\
\hline & $K^{*}(1410) \pi$ & 2.91 & 0 & - \\
\hline & $K_{1}(1270) \pi$ & 11.4 & 2 & - \\
\hline & $K_{1}(1400) \pi$ & 6.47 & 2 & - \\
\hline & $K \phi$ & 0.783 & 1 & - \\
\hline & $K a_{1}$ & 4.17 & 1 & - \\
\hline & $K a_{2}$ & 13.5 & 1 & - \\
\hline & $K b_{1}$ & 13.0 & 2 & - \\
\hline & $K^{*} \phi$ & 3.84 & 3 & - \\
\hline & $K_{2}^{*}(1430) \pi$ & 15.9 & 2 & - \\
\hline & $K_{1}(1270) \eta$ & 3.13 & 1 & - \\
\hline & Total & 250 & 98 & $198 \pm 30$ \\
\hline & $\Gamma_{K \pi} / \Gamma_{\text {Total }}$ & $8.40 \%$ & $21 \%$ & $9.9 \pm 1.2 \%[58]$ \\
\hline & $\Gamma_{K^{*} \phi} / \Gamma_{\text {Total }}$ & $1.54 \%$ & $3.1 \%$ & $1.4 \pm 0.7 \%[74]$ \\
\hline
\end{tabular}

strong decay information of these two $G$ wave states in Table 15.

As shown in Table 15, main decay modes of $K_{4}(2310)$ are $K_{4}^{*}(2045) \pi, K_{3}^{*}(1780) \pi, K \rho_{3}(1690), K a_{2}$ and $K_{2}^{*} \pi$ when $K_{4}(2310)$ is assigned to a $1 G$ state. Its total width will 
Table 13 Strong decay information of $K_{3}(2320)$ as an $2 F$ state and (predicted) $K_{3}(2075)(1 F)$ depending on their mixing angle, where $s$ and $c$ represent sine and cosine functions. The unit of the width is $\mathrm{MeV}$

\begin{tabular}{|c|c|c|}
\hline Decay channel & $K_{3}(2075)$ as $1 F$ state & $K_{3}(2320)$ as $2 F$ state \\
\hline Total & $464 c^{2}+182 c s+530 s^{2}$ & $189 c^{2}+21.2 c s+202 s^{2}$ \\
\hline$K_{3}^{*}(1780) \pi$ & $107 c^{2}+245 c s+141 s^{2}$ & $24 c^{2}+42.4 c s+21.3 s^{2}$ \\
\hline$K^{*}(1410) \pi$ & $6.93 c^{2}+0.975 c s+7.07 s^{2}$ & $20.3 c^{2}+27.2 c s+16.4 s^{2}$ \\
\hline$K \rho$ & $38.7 c^{2}+11.1 c s+40.3 s^{2}$ & $11 c^{2}+20.4 c s+13.9 s^{2}$ \\
\hline$K^{*} \pi$ & $40.5 c^{2}+4.03 c s+39.9 s^{2}$ & $10.4 c^{2}+20.2 c s+13.3 s^{2}$ \\
\hline$K a_{2}(1700)$ & 0 & $12.9 c^{2}+13.7 c s+9.98 s^{2}$ \\
\hline$K_{2}^{*}(1980) \pi$ & 0 & $11.5 c^{2}+12.3 c s+8.81 s^{2}$ \\
\hline$K \rho_{3}(1690)$ & 0 & $8.44 c^{2}+16.2 c s+8.19 s^{2}$ \\
\hline$K^{*}(1410) \rho$ & 0 & $13.5 c^{2}+9.77 s^{2}$ \\
\hline$K^{*} \rho$ & $55.7 c^{2}+43.7 s^{2}$ & $12.3 c^{2}+8.63 s^{2}$ \\
\hline$K \rho(1450)$ & $1.51 c^{2}+3.33 c s+1.99 s^{2}$ & $7.87 c^{2}+1.95 c s+8.15 s^{2}$ \\
\hline$K \omega_{3}(1670)$ & 0 & $4.04 c^{2}+7.94 c s+4.13 s^{2}$ \\
\hline$K^{*}(1410) \eta$ & $0.0642 c^{2}+0.408 c s+0.703 s^{2}$ & $0.163 c^{2}+0.557 c s+7.7 s^{2}$ \\
\hline$K \omega$ & $12.8 c^{2}+4.01 c s+13.3 s^{2}$ & $3.63 c^{2}+6.79 c s+4.61 s^{2}$ \\
\hline$K^{*} \pi(1300)$ & 0 & $3.56 c^{2}+6.55 c s+4.51 s^{2}$ \\
\hline$K_{4}^{*}(2045) \pi$ & 0 & $4.3 c^{2}+4.62 c s+1.56 s^{2}$ \\
\hline$K(1460) \rho$ & 0 & $2.41 c^{2}+5.2 c s+3.16 s^{2}$ \\
\hline$K^{*}(1680) \pi$ & $0.248 c^{2}+1.02 c s+1.05 s^{2}$ & $3.33 c^{2}+4.79 c s+1.73 s^{2}$ \\
\hline$K_{2}^{*} \rho$ & 0 & $3.96 c^{2}+1.4 c s+3.94 s^{2}$ \\
\hline$K^{*}(1410) \omega$ & 0 & $4.14 c^{2}+2.97 s^{2}$ \\
\hline$K^{*} \omega$ & $18.1 c^{2}+14.2 s^{2}$ & $4 c^{2}+2.83 s^{2}$ \\
\hline$K a_{0}(1450)$ & $1.15 c^{2}$ & $3.91 c^{2}$ \\
\hline$K^{*} a_{2}$ & 0 & $2.73 c^{2}+0.957 c s+2.7 s^{2}$ \\
\hline$K \omega(1420)$ & $0.93 c^{2}+1.94 c s+1.21 s^{2}$ & $2.89 c^{2}+0.472 c s+2.82 s^{2}$ \\
\hline$K^{*} \eta$ & $0.106 c^{2}+1.54 c s+15.4 s^{2}$ & $0.0206 c^{2}+0.429 c s+2.86 s^{2}$ \\
\hline$K a_{2}$ & $50.3 c^{2}+54.7 c s+37.3 s^{2}$ & $1.84 c^{2}+2.13 c s+0.972 s^{2}$ \\
\hline$K a_{1}$ & $24.5 s^{2}$ & $2.33 s^{2}$ \\
\hline$K b_{1}$ & $6.76 s^{2}$ & $2.07 s^{2}$ \\
\hline$K_{0}^{*}(1945) \pi$ & 0 & $1.66 c^{2}$ \\
\hline$K \phi$ & $6.29 c^{2}+10.4 c s+7.78 s^{2}$ & $0.787 c^{2}+1.33 c s+0.98 s^{2}$ \\
\hline$K_{2}^{*} \omega$ & 0 & $1.28 c^{2}+0.452 c s+1.28 s^{2}$ \\
\hline$K f_{2}$ & $20 c^{2}+14.6 s^{2}+21.9 c s$ & $1.03 c^{2}+1.05 c s+0.123 s^{2}$ \\
\hline$K_{2}^{*} \pi$ & $55.5 c^{2}+60.8 c s+40.4 s^{2}$ & $0.742 c^{2}+0.853 c s+0.366 s^{2}$ \\
\hline$K_{2}^{*} \eta$ & $8.55 c^{2}+1.11 c s+0.0976 s^{2}$ & $0.773 c^{2}+0.0199 c s+0.000205 s^{2}$ \\
\hline$K^{*} \phi$ & $3.01 c^{2}+2.04 s^{2}$ & $0.474 c^{2}+0.585 s^{2}$ \\
\hline$K^{*} \eta^{\prime}$ & $2.7 c^{2}+1.74 c s+0.309 s^{2}$ & $0.429 c^{2}+0.206 c s+0.0584 s^{2}$ \\
\hline$K_{1}(1270) \rho$ & $5.65 c^{2}+5.95 c s+5.16 s^{2}$ & $0.263 c^{2}+0.216 c s+0.268 s^{2}$ \\
\hline$K f_{1}(1420)$ & $2.93 s^{2}$ & $0.0280 c^{2}+0.366 s^{2}$ \\
\hline$K h_{1}$ & $2.89 s^{2}$ & $0.198 c^{2}+0.347 s^{2}$ \\
\hline$K f_{1}$ & $6.98 s^{2}$ & $0.136 c^{2}+0.225 s^{2}$ \\
\hline
\end{tabular}


Table 14 The main strong decay widths of $K_{3}(2360)$ assigned to an $2 F^{\prime}$ state, where $s$ and $c$ represent sine and cosine functions. The unit of the width is $\mathrm{MeV}$

\begin{tabular}{ll}
\hline Decay channel & Width \\
\hline Total & $93 c^{2}+34 c s+101 s^{2}$ \\
$K_{3}{ }^{*}(1780) \pi$ & $28.3 c^{2}+54.7 c s+30.4 s^{2}$ \\
$\mathrm{~K} \rho_{3}(1690)$ & $16 c^{2}-22.8 c s+22.8 s^{2}$ \\
$\mathrm{~K} \rho$ & $15.1 c^{2}-20.3 c s+12.1 s^{2}$ \\
$K^{*} \pi$ & $14.3 c^{2}+20 c s+11.4 s^{2}$ \\
$K_{4}{ }^{*}(2045) \pi$ & $2.52 c^{2}+11.9 c s+11.4 s^{2}$ \\
$\mathrm{~K} \omega_{3}(1670)$ & $6.88 c^{2}-9.62 c s+9.62 s^{2}$ \\
$K^{*} \rho$ & $9.83 c^{2}+14.3 s^{2}$ \\
\hline
\end{tabular}

be (710-880) MeV, which is not easy to observe in experiments.

\subsection{2 $2 G$ states}

$K_{4}(2500)$ and its partner $K_{4}(2550)$ (predicted) satisfy

$\left(\begin{array}{l}\left|K_{4}(2500)\right\rangle \\ \left|K_{4}(2550)\right\rangle\end{array}\right) \approx\left(\begin{array}{cc}\cos \theta_{2 G} & \sin \theta_{2 G} \\ -\sin \theta_{2 G} & \cos \theta_{2 G}\end{array}\right)\left(\begin{array}{l}\left|2^{1} G_{4}\right\rangle \\ \left|2^{3} G_{4}\right\rangle\end{array}\right)$,

where $K_{4}(2550)$ is obtained from an equation similar to Eq. (42).

The total width of $K_{4}(2500)$ assigned to a $2 G$ state is about (230-290) MeV, which is consistent with the experimental value $\sim 250 \mathrm{MeV}$ [76]. According to Table 15, the main decay channels of $K_{4}(2500)$ are $K_{3}^{*}(1780) \pi$, $K \rho_{3}(1690), K a_{2}, K_{2}^{*} \pi, K_{4}^{*}(2045) \pi, K^{*} b_{1}, K_{2}^{*}(1980) \pi$ and $K \omega_{3}(1670)$, etc. Information of these predicted decay widths is important to study the mixing angle of this state for future experiments.

The total width of $K_{4}(2550)$ assigned to a $2 G^{\prime}$ state is about (230-260) MeV. According to Table 16, the main decay channels of $K_{4}(2550)$ are $K_{3}^{*}(1780) \pi, K \rho_{3}(1690)$, $K a_{2}, K_{4}^{*}(2045) \pi, K_{2}^{*}(1980) \pi$ and $K^{*} b_{1}$, etc. We hope our prediction can be helpful for the future experiment to study these two states and their mixing angle.

\section{Conclusions and discussion}

In this paper, we have given the analysis of the mass spectra of the kaon family via the modified Godfrey-Isgur quark model that includes a color screening effect, and we have obtained the structure information of the observed kaon candidates. Then we have further tested the possible assignments by comparing the theoretical results of their two-body strong decays with the experimental data. Additionally, we have also predicted the behaviors of some partial decay widths of the
Table 15 The widths of (predicted) $K_{4}(2310)$ and $K_{4}$ (2500) depending on their mixing angle, where $s$ and $c$ represent sine and cosine functions. The unit of the width is $\mathrm{MeV}$

\begin{tabular}{|c|c|c|}
\hline $\begin{array}{l}\text { Decay } \\
\text { channels }\end{array}$ & $\begin{array}{l}\text { Width }\left(K_{4}(2310)\right. \\
\text { assigned to } 1 G \text { state })\end{array}$ & $\begin{array}{l}\text { Width }\left(K_{4}(2500)\right. \\
\text { assigned to } 2 G \text { state })\end{array}$ \\
\hline Total & $664 c^{2}+31 c s+754 s^{2}$ & $222 c^{2}+7.5 c s+247 s^{2}$ \\
\hline$K_{4}^{*}(2045) \pi$ & $90.2 c^{2}+201 c s+112 s^{2}$ & $14.6 c^{2}+28.7 c s+14.6 s^{2}$ \\
\hline$K_{3}^{*}(1780) \pi$ & $80 c^{2}+168 c s+92.3 s^{2}$ & $21.6 c^{2}+44.1 c s+24.7 s^{2}$ \\
\hline$K \rho_{3}(1690)$ & $63.1 c^{2}+140 . c s+77.6 s^{2}$ & $19 c^{2}+41.6 c s+23.3 s^{2}$ \\
\hline $\mathrm{Ka}_{2}$ & $43.1 c^{2}+70.4 c s+40.9 s^{2}$ & $17.5 c^{2}+34 . c s+20.5 s^{2}$ \\
\hline$K_{2}^{*} \pi$ & $41.7 c^{2}+62.6 c s+38.6 s^{2}$ & $16.7 c^{2}+31.7 c s+20.2 s^{2}$ \\
\hline$K \omega_{3}(1670)$ & $24 c^{2}+52.8 c s+29.2 s^{2}$ & $7.11 c^{2}+15.5 c s+8.69 s^{2}$ \\
\hline$K^{*} b_{1}$ & $22.2 c^{2}+45.2 c s+27.1 s^{2}$ & $8.14 c^{2}+17.2 c s+10 . s^{2}$ \\
\hline$K^{*} \rho$ & $29.9 c^{2}+28.2 s^{2}$ & $9.16 c^{2}+7.28 s^{2}$ \\
\hline$K^{*} \pi$ & $19 c^{2}+15 c s+17.3 s^{2}$ & $7.05 c^{2}+3.28 c s+7.42 s^{2}$ \\
\hline$K f_{2}$ & $15.2 c^{2}+22.3 c s+13.3 s^{2}$ & $6.22 c^{2}+11.7 c s+7.08 s^{2}$ \\
\hline$K^{*} a_{1}$ & $10.9 c^{2}+22.5 c s+12.9 s^{2}$ & $4.18 c^{2}+8.74 c s+5.1 s^{2}$ \\
\hline$K^{*} h_{1}$ & $10.8 c^{2}+20.2 c s+12.9 s^{2}$ & $3.74 c^{2}+7.88 c s+4.6 s^{2}$ \\
\hline$K \rho$ & $17.5 c^{2}+7.35 c s+16.7 s^{2}$ & $7.76 c^{2}+4 . c s+8.21 s^{2}$ \\
\hline$K^{*} a_{2}$ & $15.4 c^{2}+11.5 c s+9.12 s^{2}$ & $5.58 c^{2}+3.68 c s+4.01 s^{2}$ \\
\hline$K_{2}^{*} \rho$ & $14.9 c^{2}+11.2 c s+8.83 s^{2}$ & $3.36 c^{2}+2.39 c s+2.96 s^{2}$ \\
\hline$K_{2}^{*} \eta$ & $0.241 c^{2}+3.62 c s+17.1 s^{2}$ & $0.139 c^{2}+0.96 c s+2.35 s^{2}$ \\
\hline$K^{*}(1410) \pi$ & $10.2 c^{2}+7.29 c s+9.41 s^{2}$ & $4.39 c^{2}+7.83 c s+3.51 s^{2}$ \\
\hline$K a_{1}$ & $3.07 c^{2}+11.1 c s+9.99 s^{2}$ & $1.19 c^{2}+2.63 c s+1.46 s^{2}$ \\
\hline$K^{*} f_{2}$ & $9.84 c^{2}+7.37 c s+5.98 s^{2}$ & $3.04 c^{2}+1.99 c s+2.07 s^{2}$ \\
\hline$K^{*} \omega$ & $9.79 c^{2}+9.18 s^{2}$ & $3.0 c^{2}+2.38 s^{2}$ \\
\hline$K \rho(1450)$ & $5.22 c^{2}+7.23 c s+6.02 s^{2}$ & $0.95 c^{2}+1.67 c s+0.764 s^{2}$ \\
\hline$K \omega$ & $5.77 c^{2}+2.3 c s+5.51 s^{2}$ & $2.55 c^{2}+1.38 c s+2.71 s^{2}$ \\
\hline$K^{*}(1680) \pi$ & $2.31 c^{2}+6.41 c s+4.45 s^{2}$ & $0.439 c^{2}+0.2 c s+0.0385 s^{2}$ \\
\hline$K_{2}^{*} \omega$ & $4.35 c^{2}+3.26 c s+2.57 s^{2}$ & $0.01 c^{2}+0.724 c s+0.909 s^{2}$ \\
\hline$K_{0}^{*}(1430) \pi$ & $2.3 c^{2}+5.12 c s+2.84 s^{2}$ & $0.0247 c^{2}+0.232 c s+0.546 s^{2}$ \\
\hline$K_{2}^{*}(1980) \pi$ & $1.43 c^{2}+3.14 c s+1.76 s^{2}$ & $9.02 c^{2}+16.7 c s+9.55 s^{2}$ \\
\hline$K \phi$ & $1.92 c^{2}+2.01 c s+2.14 s^{2}$ & $0.36 c^{2}+0.771 c s+0.446 s^{2}$ \\
\hline$K b_{1}$ & $3.02 s^{2}$ & $0.988 s^{2}$ \\
\hline$K^{*} \phi$ & $2.08 c^{2}+1.67 s^{2}$ & $0.247 c^{2}+0.198 s^{2}$ \\
\hline$K^{*}(1410) \eta$ & $1.4 c^{2}+0.694 c s+0.209 s^{2}$ & $1.82 c^{2}+0.611 c s+0.0524 s^{2}$ \\
\hline$K^{*}(1410) \rho$ & $1.03 c^{2}+0.79 s^{2}$ & $6.55 c^{2}+6.31 s^{2}$ \\
\hline
\end{tabular}

kaons, which are still missing in experiments. In Table 17, we summarize the mass and main decay modes of these states, by which experiment may carry out the search for them.

This study is crucial to establish the kaon family and future search for their higher excitations. We have discussed the possible assignments to the kaons listed in PDG. The main task of the present work has been a calculation of the spectra and OZI-allowed two-body strong decays of the kaon family, which can test the possible assignments to the kaons. In 
Table 16 The strong decay widths of $K_{4}(2550)$ assigned to a $2 G^{\prime}$ state, where $s$ and $c$ represent sine and cosine functions. The unit of the width is $\mathrm{MeV}$

\begin{tabular}{ll}
\hline Decay channel & Width \\
\hline Total & $238 c^{2}+38 c s+218 s^{2}$ \\
$K_{3}^{*}(1780) \pi$ & $32.7 c^{2}+57.5 c s+28.3 s^{2}$ \\
$K \rho_{3}(1690)$ & $31.2 c^{2}-55.3 c s+25.7 s^{2}$ \\
$K_{4}^{*}(2045) \pi$ & $25.1 c^{2}+48.1 c s+24 s^{2}$ \\
$K a_{2}$ & $21.8 c^{2}-35.8 c s+19.8 s^{2}$ \\
$K_{2}^{*} \pi$ & $23 c^{2}+34.8 c s+19.4 s^{2}$ \\
$K^{*} b_{1}$ & $15.1 c^{2}+25.8 c s+12.3 s^{2}$ \\
$K \omega_{3}(1670)$ & $10.9 c^{2}-19.3 c s+9.05 s^{2}$ \\
$K \rho$ & $9.72 c^{2}-1.24 c s+9.58 s^{2}$ \\
$K^{*} a_{2}$ & $7.94 c^{2}-7.73 c s+11.9 s^{2}$ \\
$K^{*} a_{1}$ & $5.90 c^{2}+6.60 c s+4.95 s^{2}$ \\
$K_{2}^{*} \rho$ & $6.29 c^{2}+5.64 c s+8.34 s^{2}$ \\
\hline
\end{tabular}

Table 17 The mass and the important strong decay channels for some predicted kaon states which can be helpful in future search for them in experiments. The units of the mass and width are $\mathrm{MeV}$

\begin{tabular}{llll}
\hline State & Assignment & Mass & Main decay channels \\
\hline$K_{1}(2030)$ & $2 P^{\prime}$ & $\sim 2030$ & $K^{*} \pi, K \rho$ \\
$K_{2}(1990)$ & $2 D$ & $\sim 1994$ & $K^{*} \pi, K \rho$ \\
$K_{3}(2075)$ & $1 F$ & $\sim 2075$ & $K_{3}^{*}(1780) \pi, K^{*} \rho$ \\
$K_{3}(2360)$ & $2 F^{\prime}$ & $\sim 2362$ & $K_{3}^{*}(1780) \pi, K \rho_{3}(1690)$ \\
$K_{4}(2310)$ & $1 G$ & $\sim 2309$ & $K_{4}^{*}(2045) \pi, K_{3}^{*}(1780) \pi$ \\
$K_{4}(2550)$ & $2 G^{\prime}$ & $\sim 2550$ & $K_{3}^{*}(1780) \pi, K \rho_{3}(1690)$ \\
\hline
\end{tabular}

Sects. 2 and 3, we have discussed these points in detail. The predicted decay behaviors of the discussed kaons can provide valuable information for further experimental study in the future.

At present, experimental information on the kaons is not abundant. Thus, we suggest to do more experimental measurements of the resonance parameters and to search for the missing main decay channels. Such an effort will be not only helpful to establish the kaon family in experiments, but it is also valuable to study the production of hiddencharm pentaquarks $P_{c}(4380)$ and $P_{c}(4450)$ by analyzing $\Lambda_{b} \rightarrow J / \psi p K$ [77], which has a close relation to the understanding of the kaon family. With experimental progress, the exploration of the kaons will become a major issue in hadron physics, we have been provided good platforms in the BESIII, BelleII, and COMPASS experiments. We hope that, inspired by this work, more experimental and theoretical studies of high-spin states are conducted in the future.

Acknowledgements This work is supported in part by National Natural Science Foundation of China under the Grant No. 11222547 and No. 11175073, the Fundamental Research Funds for the Central Uni- versities, the High-End Creative Talent Thousand People Plan of Qinghai Province, No. 0042801 and the Applied Basic Research Project of Qinghai Province, No. 2017-ZJ-748. Xiang Liu is also supported by the National Program for Support of Top-notch Young Professionals.

Open Access This article is distributed under the terms of the Creative Commons Attribution 4.0 International License (http://creativecomm ons.org/licenses/by/4.0/), which permits unrestricted use, distribution, and reproduction in any medium, provided you give appropriate credit to the original author(s) and the source, provide a link to the Creative Commons license, and indicate if changes were made.

Funded by SCOAP ${ }^{3}$.

\section{References}

1. K.A. Olive et al., Particle Data Group. Chin. Phys. C 38, 090001 (2014)

2. S. Godfrey, N. Isgur, Phys. Rev. D 32, 189 (1985)

3. T. Barnes, N. Black, P.R. Page, Phys. Rev. D 68, 054014 (2003). arXiv:nucl-th/0208072 [nucl-th]

4. D. Ebert, R. Faustov, V. Galkin, Phys. Rev. D 79, 114029 (2009). arXiv:0903.5183 [hep-ph]

5. Q.-T. Song, D.-Y. Chen, X. Liu, T. Matsuki, Phys. Rev. D 91, 054031 (2015a). arXiv:1501.03575 [hep-ph]

6. Q.-T. Song, D.-Y. Chen, X. Liu, T. Matsuki, Phys. Rev. D 92, 074011 (2015b). arXiv:1503.05728 [hep-ph]

7. L. Micu, Nucl. Phys. B 10, 521 (1969)

8. A. Le Yaouanc, L. Oliver, O. Pene, J. Raynal, Phys. Rev. D 8, 2223 (1973)

9. E. van Beveren, C. Dullemond, G. Rupp, Phys. Rev. D 21, 772 (1980) [Erratum: Phys. Rev. D 22, 787 (1980)]

10. E. van Beveren, G. Rupp, T. Rijken, C. Dullemond, Phys. Rev. D 27, 1527 (1983)

11. A. Le Yaouanc, L. Oliver, O. Pene, J.C. Raynal, Hardron Transitions In The Quark Model (Gordon and Breach, New York, 1988)

12. W. Robertsand, B. Silvestre-Brac, Acta Phys. Austriaca 11, 171 (1992)

13. S. Capstickand, W. Roberts, Phys. Rev. D 49, 4570 (1994). arXiv:nucl-th/9310030 [nucl-th]

14. H.G. Blundelland, S. Godfrey, Phys. Rev. D 53, 3700 (1996). arXiv:hep-ph/9508264 [hep-ph]

15. E. Ackleh, T. Barnes, E. Swanson, Phys. Rev. D 54, 6811 (1996). arXiv:hep-ph/9604355 [hep-ph]

16. S. Capstickand, B. Keister. arXiv:nucl-th/9611055 [nucl-th]

17. R. Bonnaz, B. Silvestre-Brac, C. Gignoux, Eur. Phys. J. A 13, 363 (2002). arXiv:hep-ph/0101112 [hep-ph]

18. F. Closeand, E. Swanson, Phys. Rev. D 72, 094004 (2005). arXiv:hep-ph/0505206 [hep-ph]

19. B. Zhang, X. Liu, W.-Z. Deng, S.-L. Zhu, Eur. Phys. J. C 50, 617 (2007). arXiv:hep-ph/0609013 [hep-ph]

20. J. Lu, X.-L. Chen, W.-Z. Deng, S.-L. Zhu, Phys. Rev. D 73, 054012 (2006). arXiv:hep-ph/0602167 [hep-ph]

21. Z.-F. Sunand, X. Liu, Phys. Rev. D 80, 074037 (2009). arXiv:0909.1658 [hep-ph]

22. X. Liu, Z.-G. Luo, Z.-F. Sun, Phys. Rev. Lett. 104, 122001 (2010). arXiv:0911.3694 [hep-ph]

23. Z.-F. Sun, J.-S. Yu, X. Liu, T. Matsuki, Phys. Rev. D 82, 111501 (2010). arXiv:1008.3120 [hep-ph]

24. T. Rijken, M. Nagels, Y. Yamamoto, Nucl. Phys. A 835, 160 (2010)

25. J.-S. Yu, Z.-F. Sun, X. Liu, Q. Zhao, Phys. Rev. D 83, 114007 (2011). arXiv:1104.3064 [hep-ph]

26. Z.-Y. Zhouand, Z. Xiao, Phys. Rev. D 84, 034023 (2011). arXiv:1105.6025 [hep-ph] 
27. Z.-C. Ye, X. Wang, X. Liu, Q. Zhao, Phys. Rev. D 86, 054025 (2012). arXiv:1206.0097 [hep-ph]

28. X. Wang, Z.-F. Sun, D.-Y. Chen, X. Liu, T. Matsuki, Phys. Rev. D 85, 074024 (2012). arXiv:1202.4139 [hep-ph]

29. Y. Sun, X. Liu, T. Matsuki, Phys. Rev. D 88, 094020 (2013). arXiv:1309.2203 [hep-ph]

30. L.-P. He, X. Wang, X. Liu, Phys. Rev. D 88, 034008 (2013). arXiv:1306.5562 [hep-ph]

31. Y. Sun, Q.-T. Song, D.-Y. Chen, X. Liu, S.-L. Zhu, Phys. Rev. D 89, 054026 (2014). arXiv:1401.1595 [hep-ph]

32. C.-Q. Pang, L.-P. He, X. Liu, T. Matsuki, Phys. Rev. D 90, 014001 (2014). arXiv:1405.3189 [hep-ph]

33. B. Wang, C.-Q. Pang, X. Liu, T. Matsuki, Phys. Rev. D 91, 014025 (2015). arXiv:1410.3930 [hep-ph]

34. K. Chen, C.-Q. Pang, X. Liu, T. Matsuki, Phys. Rev. D 91, 074025 (2015). arXiv:1501.07766 [hep-ph]

35. C. Langand, C. Rebbi, Phys. Lett. B 115, 137 (1982)

36. S. Booth, D. Henty, A. Hulsebos, A. Irving, C. Michael, P. Stephenson, U. Collaboration et al., Phys. Lett. B 294, 385 (1992)

37. G.S. Bali, Phys. Rept. 343, 1 (2001)

38. T. Kawanaiand, S. Sasaki, Phy. Rev. Lett 107, 091601 (2011)

39. T. Kawanaiand, S. Sasaki, Prog. Part. Nuclear Phys. 67, 130 (2012)

40. W. Lucha, F.F. Schoberl, D. Gromes, Phys. Rept. 200, 127 (1991)

41. A. Le Yaouanc, L. Oliver, O. Pene, J.-C. Raynal, Phys. Rev. D 9, 1415 (1974)

42. A. Le Yaouanc, L. Oliver, O. Pene, J. Raynal, Phys. Rev. D 11, $1272(1975)$

43. A. Le Yaouanc, L. Oliver, O. Pene, J. Raynal, Phys. Lett. B 72, 57 (1977a)

44. A. Le Yaouanc, L. Oliver, O. Pene, J.-C. Raynal, Phys. Lett. B 71, 397 (1977b)

45. P.R. Page, Nucl. Phys. B. 446, 189 (1995). arXiv:hep-ph/9502204 [hep-ph]

46. A.I. Titov, T.I. Gulamov, B. Kampfer, Phys. Rev. D 53, 3770 (1996)

47. H.G. Blundell. arXiv:hep-ph/9608473 (1996)

48. H.Q. Zhou, R.G. Ping, B.S. Zou, Phys. Lett. B 611, 123 (2005). arXiv:hep-ph/0412221 [hep-ph]

49. Z.-G. Luo, X.-L. Chen, X. Liu, Phys. Rev. D 79, 074020 (2009). arXiv:0901.0505 [hep-ph]

50. M. Jacoband, G. Wick, Ann. Phys. 7, 404 (1959)

51. B.-Q. Liand, K.-T. Chao, Commun. Theor. Phys. 52, 653 (2009a). arXiv:0909.1369 [hep-ph]

52. B.-Q. Liand, K.-T. Chao, Phys. Rev. D 79, 094004 (2009b). arXiv:0903.5506 [hep-ph]

53. T. Matsuki, T. Morii, K. Seo, Prog. Theor. Phys. 124, 285 (2010). arXiv:1001.4248 [hep-ph]
54. G.D. Tikhomirov, I.A. Erofeev, O.N. Erofeeva, V.N. Luzin, Phys. Atom. Nucl. 66, 828 (2003) [Yad. Fiz. 66, 860 (2003)]

55. C. Daum et al., ACCMOR. Nucl. Phys. B 187, 1 (1981)

56. G.W. Brandenburg, R.K. Carnegie, R.J. Cashmore, M. Davier, W.M. Dunwoodie, T.A. Lasinski, D.W.G.S. Leith, J.A.J. Matthews, P. Walden, S.H. Williams, Phys. Rev. Lett. 36, 1239 (1976)

57. R. Aaij et al., LHCb. Phys. Rev. D 93, 052018 (2016). arXiv:1509.06628 [hep-ex]

58. D. Aston et al., Nucl. Phys. B 296, 493 (1988a)

59. D. Antreasyan et al., Crystal Ball. Z. Phys. C 48, 561 (1990)

60. R.K. Carnegie, R.J. Cashmore, M. Davier, W.M. Dunwoodie, T.A. Lasinski, D.W.G.S. Leith, S.H. Williams, Nucl. Phys. B 127, 509 (1977)

61. H.-Y. Cheng, C.-K. Chua, C.-W. Hwang, Phys. Rev. D 69, 074025 (2004). arXiv:hep-ph/0310359 [hep-ph]

62. D.-M. Liand, Z. Li, Eur. Phys. J. A 28, 369 (2006). arXiv:hep-ph/0606297 [hep-ph]

63. A. Tayduganov, E. Kou, A. Le Yaouanc, Phys. Rev. D 85, 074011 (2012). arXiv:1111.6307 [hep-ph]

64. D. Aston et al., Nucl. Phys. B 292, 693 (1987)

65. D. Frame, I.S. Hughes, J.G. Lynch, P.W. Minto, D.M. McFadzean, D.T. Stewart, A.S. Thompson, R.M. Turnbull, I.T. Wilkie, Nucl. Phys. B 276, 667 (1986)

66. R. Aaij et al., LHCb. Phys. Rev. Lett. 118, 022003 (2017). arXiv:1606.07895 [hep-ex]

67. T. Armstrong et al., Bari-Birmingham-CERN-Milan-Paris-Pavia. Nucl. Phys. B 221, 1 (1983a)

68. H.-Y. Cheng, Phys. Lett. B 707, 116 (2012). arXiv:1110.2249 [hep$\mathrm{ph}]$

69. A. Firestone, G. Goldhaber, D. Lissauer, G.H. Trilling, Phys. Rev. D 5, 505 (1972)

70. M. Baubillier et al., Birmingham-CERN-Glasgow-Michigan StateParis. Z. Phys. C 26, 37 (1984)

71. D. Aston et al., Phys. Lett. B 149, 258 (1984)

72. D. Aston et al., Phys. Lett. B 201, 169 (1988b)

73. D. Aston et al., Phys. Lett. B 99, 502 (1981)

74. D. Aston et al., Phys. Lett. B. 180, 308 (1986) [Erratum: Phys. Lett. B 183, 434 (1987)]

75. T. Armstrong et al., Bari-Birmingham-CERN-Milan-Paris-Pavia. Nucl. Phys. B 227, 365 (1983b)

76. W.E. Cleland et al., Nucl. Phys. B 184, 1 (1981)

77. R. Aaij et al., LHCb. Phys. Rev. Lett. 115, 072001 (2015). arXiv:1507.03414 [hep-ex] 